\title{
REPRESENTATIONS OF CROSSED PRODUCTS BY COACTIONS AND PRINCIPAL BUNDLES
}

\author{
M. B. LANDSTAD, J. PHILLIPS, I. RAEBURN \\ AND C. E. SUTHERLAND
}

\begin{abstract}
The main purpose of this paper is to establish a covariant representation theory for coactions of locally compact groups on $C^{*}$-algebras (including a notion of exterior equivalence), to show how these results extend the usual notions for actions of groups on $C^{*}$-algebras, and to apply these ideas to classes of coactions termed pointwise unitary and locally unitary to obtain a complete realization of the isomorphism theory of locally trivial principal $G$-bundles in this context. We are also able to obtain all (Cartan) principal $G$-bundles in this context, but their isomorphism theory remains elusive.
\end{abstract}

Introduction. The main purpose of this paper is to establish a covariant representation theory for coactions of locally compact groups on $C^{*}$-algebras (including a notion of exterior equivalence), to show how these results extend the usual notions for actions of groups on $C^{*}$-algebras, and to apply these ideas to classes of coactions termed pointwise unitary and locally unitary to obtain a complete realization of the isomorphism theory of locally trivial principal $G$-bundles in this context. We are also able to obtain all (Cartan) principal $G$-bundles in this context, but their isomorphism theory remains elusive.

The notion of an action $\alpha$ of a group $G$ on a $C^{*}$-algebra $A$ is well known and widely discussed in the literature, and the analysis of the crossed product $A \times{ }_{\alpha} G$ and its representations has been a focal point in recent research. The dual notion of coaction is much less well known, and has been used principally to formulate and prove duality theorems in the context of nonabelian groups; see $[10,12,13,19]$ for $C^{*}$-algebraic aspects and [14] for what is known of coactions on von Neumann algebras.

A coaction $\delta$ of $G$ on $A$ may be thought of in many ways. The (rather technical) definition is given in the preliminary section, but if $A$ is a unital $C^{*}$-algebra and $G$ is finite, it is a unital injective *-homomorphism $\delta: A \rightarrow A \otimes \mathbf{C}(G)$ (where $\mathrm{C}(G)$ is the complex group algebra of $G$ ) which satisfies

$$
(\delta \otimes i) \circ \delta=\left(i \otimes \delta_{G}\right) \circ \delta,
$$

where $\delta_{G}: \mathbf{C}(G) \rightarrow \mathbf{C}(G) \otimes \mathbf{C}(G)$ is the map determined by $\delta_{G}(\lambda(s))=\lambda(s) \otimes \lambda(s)$ for $s \in G$ (where $\lambda(s)$ is the element in $\mathbf{C}(G)$ corresponding to $s \in G$ ). Note that $\delta_{G}$

Received by the editors March 25, 1985.

1980 Mathematics Subject Classification. Primary 46L40, 46L55, 46M20; Secondary 55R10. 
is itself a coaction of $G$ on $\mathbf{C}(G)$. If we write

$$
\delta(a)=\sum_{s \in G} a(s) \otimes \lambda(s) \quad \text { for } a \in A,
$$

the identity (1) tells us that

$$
\sum_{s \in G} \delta(a(s)) \otimes \lambda(s)=\sum_{s \in G} a(s) \otimes \lambda(s) \otimes \lambda(s)
$$

so that $a(s) \in A_{s}=\{a \in A \mid \delta(a)=a \otimes \lambda(s)\}$. Furthermore since

$$
\delta(a)=\sum_{s \in G} a(s) \otimes \lambda(s)=\sum_{s \in G} \delta(a(s))=\delta\left(\sum_{s \in G} a(s)\right)
$$

and $\delta$ is injective we obtain $a=\sum_{s \in G} a(s)$ and $A=\oplus_{s \in G} A_{s}$. In addition, it is clear that $A_{s}^{*}=A_{s-1}$ and $A_{s} A_{t} \subseteq A_{s t}$ for $s, t \in G$. Conversely, if we are given a $C^{*}$-algebra $A$ and closed subspaces $\left\{A_{s}\right\}_{s \in G}$ with $A_{s}^{*}=A_{s}^{-1}, A_{s} A_{t} \subseteq A_{s t}$ and $A=\bigoplus_{s \in G} A_{s}$ (in which case we say $A$ is $G^{-*}$-graded by $\left\{A_{s}\right\}$ ), we may define a coaction $\delta$ of $G$ on $A$ by the condition $\delta(a)=a \otimes \lambda(s)$ for $a \in A_{s}$. Thus, for unital $A$ and finite $G$, coactions of $G$ on $A$ and $G$ - $^{*}$-gradings of $A$ are the same (this was known to Fell).

Further insight into the nature of a general coaction may be derived from consideration of abelian groups. If $\hat{G}$ is a locally compact abelian group with dual group $G$ and $\alpha$ is an action of $\hat{G}$ on $A$, then $\alpha$ defines a map $\tilde{\alpha}$ from $A$ to $C_{b}(\hat{G}, A)$ via $(\tilde{\alpha}(a))(\gamma)=\alpha_{\gamma}(a), a \in A, \gamma \in \hat{G}$. The fact that $\alpha_{\gamma_{1}} \circ \alpha_{\gamma_{2}}=\alpha_{\gamma_{1} \gamma_{2}}$ for $\gamma_{1}, \gamma_{2} \in \hat{G}$ says that

$$
(\tilde{\alpha} \otimes i) \circ \tilde{\alpha}=\left(i \otimes \alpha_{G}\right) \circ \tilde{\alpha},
$$

where $\alpha_{G}: C_{b}(\hat{G}) \rightarrow C_{b}(\hat{G} \times \hat{G})$ is given by $\left(\alpha_{G} f\right)\left(\gamma_{1}, \gamma_{2}\right)=f\left(\gamma_{1} \gamma_{2}\right)$. If we recall that the Fourier transform maps $C_{b}(\hat{G})$ to the multiplier algebra $M\left(C_{r}^{*}(G)\right)$ of the (reduced) group $C^{*}$-algebra $C_{r}^{*}(G)$ of $G$, and that the Fourier transform of $\alpha_{G}$ is (the nondiscrete analogue of) $\delta_{G}$, then we see that equation (1) is just the Fourier transform of equation (2). Thus for locally compact abelian $G$, a coaction of $G$ on $A$ is effectively the same as an action of the dual group $\hat{G}$ on $A$. This correspondence often allows one to translate notions dealing with actions to notions dealing with coactions.

In this second example, we see that integrating the action of $\hat{G}$ gives a representation of $L^{1}(\hat{G})$ as operators on $A$ and if we take the Fourier transform we get a representation of $A(G)$, the Fourier algebra, as operators on $A$. In the case of a general coaction, one can still show by using slice maps that we get a representation of $A(G)$ as operators on $A$ : it would be esthetically pleasing and probably very useful to have some sort of intrinsic characterization of representations of $A(G)$ on $A$ which give rise to coactions of $G$ on $A$. We are unaware of any such condition, however.

This line of thought does hold some promise however. For, if $\delta$ is a coaction of $G$ on $A, \varepsilon: A(G) \rightarrow \mathscr{B}(A)$ is the corresponding representation of $A(G)$, and $(\pi, \rho)$ is a pair of representations of $(A, A(G))$ on a Hilbert space $H$, then from [14, Theorem A.1(b)] one can obtain by using slice maps a representation $\varepsilon_{\rho}$ of $A(G)$ as bounded 
operators on $\mathscr{B}(H)$, i.e., a homomorphism $\varepsilon_{\rho}: A(G) \rightarrow \mathscr{B}(\mathscr{B}(H))$. The condition we obtain in $\S 3$ for the pair $(\pi, \rho)$ to be covariant can easily be translated into the equation:

$$
\pi(\varepsilon(f)(a))=\varepsilon_{\rho}(f)(\pi(a)), \quad a \in A, f \in A(G) .
$$

In the case of abelian $G$ this is exactly (the Fourier transform of) the integrated version of the usual covariance condition! Unfortunately, until someone obtains an analogue of the Banach *-algebra $L^{1}(G, A, \alpha)$ in the coaction setting, these will remain as merely tantalizing observations. The lack of such a Banach *-algebra whose representations correspond bijectively to covariant pairs of representations created serious difficulties for us and necessitated the development of techniques quite different from those commonly used in the study of actions.

In the preliminary section we assemble certain technical tools which will have repeated use in the paper. These results concern factorization, multiplier algebras, tensor products, slice maps, and the regular representations of a locally compact group.

In $\$ 2$ we define coactions and the technical nondegeneracy condition. We provide numerous examples, of which the "dual" coactions and the "unitary" coactions play the most important role in later sections. We then define the crossed product $A \times{ }_{\delta} G$ of the $C^{*}$-algebra $A$ by the coaction $\delta$ of the locally compact group $G$. This is done in a "space-free" fashion, in that we do not use any concrete representation of $A$ on a Hilbert space. We proceed to the definition of exterior equivalence of coactions and prove the expected (but not trivial) result that exterior equivalent coactions give rise to isomorphic crossed products. As an immediate corollary, we deduce Quigg's result [19] that if $\delta$ is a unitary coaction, then $A \times{ }_{\delta} G \cong A \otimes C_{0}(G)$.

In $\$ 3$ we turn to representation theory. The main result of this section gives a natural bijection between (nondegenerate) representations of the crossed product $A \times{ }_{\delta} G$ on a Hilbert space $H$ and covariant pairs of (nondegenerate) representations on $H$ of the algebras, $A, A(G)$. This is a key result allowing one to analyze representations of $A \times{ }_{\delta} G$ : although the result is completely analogous to the action case, the proof is completely different and considerably more difficult. We use this result to show how covariant pairs transform under an exterior equivalence isomorphism.

In $\S 4$ we present the rather technical definition that an ideal $I$ in $A$ be invariant under a coaction $\delta$. For amenable $G$, at least, this turns out to be the same as requiring $I$ to be invariant under the corresponding representation $\varepsilon$ of $A(G)$ on $A$. In general, we show that if $I$ is an ideal in $A$ invariant under $\delta$ then we can identify $I \times{ }_{\delta \mid t} G$ as an ideal in $A \times{ }_{\delta} G$. In case $G$ is amenable we show that $\delta$ induces a coaction $\delta^{I}$ of $G$ on $A / I$ and that the natural surjection of $A \times{ }_{\delta} G$ onto $(A / I) \times{ }_{\delta^{\prime}} G$ induces the expected isomorphism $A \times{ }_{\delta} G / I \times{ }_{\delta} G \cong(A / I) \times{ }_{\delta^{\prime}} G$. In the remarks in $\S 4$ we point out the technical difficulties which necessitated the assumption that $G$ be amenable for the general theorems. This is important in $\S \S 5$ and 6 where we are dealing with special types of coactions and can show that the difficulties disappear in these cases. Thus, the hypothesis that $G$ be amenable is not necessary in $\S \S 5$ and 6. 
We define the notions of pointwise unitary and locally unitary coactions in $\S 5$ and show that for such coactions the spectrum of $A \times{ }_{\delta} G$ is fibered over $\hat{A}$ by the dual action of $G$. If the coaction is locally unitary this fibration is a locally trivial principal $G$-bundle. In the locally unitary case we show that two such coactions are exterior equivalent if and only if the corresponding $G$-bundles are isomorphic. Finally in $\$ 5$ we use duality theory to show how to construct examples of the two types of coactions.

In $\S 6$ we show how to construct coactions so that $p:\left(A \times{ }_{\delta} G\right)^{\wedge} \rightarrow \hat{A}$ is isomorphic to any given (Cartan) principal $G$-bundle. In fact, if $A$ is a stable $C^{*}$-algebra with paracompact, Hausdorff spectrum $T$ and $q: E \rightarrow T$ is any (Cartan) principal $G$-bundle over $T$ we construct a pointwise unitary coaction $\delta$ of $G$ on $A$ so that $p$ : $\left(A \times{ }_{\delta} G\right)^{\wedge} \rightarrow \hat{A}=T$ is isomorphic to $q: E \rightarrow T$. If $q: E \rightarrow T$ is locally trivial we can choose $\delta$ to be locally unitary and then $\delta$ is unique up to exterior equivalence.

This work was begun in January 1982, while the last three-named authors were visiting the Australian National University; thanks are due to Professor D. W. Robinson for his hospitality and financial support during this period. The first-named author would like to thank U. N. S. W. for its hospitality and financial support during his visit to U. N. S. W. in November 1983, at which time substantial progress was made and a preliminary draft of some of these results was written up. The second-named author would like to thank U. N. S. W., N. S. E. R. C. of Canada, and the University of Victoria for financial support at various times during work on this paper. All four authors are indebted to the A. R. G. S. for financial support during the preparation of this paper.

1. Preliminaries. On many occasions it will be important, in a calculation, to factor elements in a Banach space with elements from certain $C^{*}$-algebras. In most cases, an approximate factorization will suffice, provided we mumble politely about continuity. However, to avoid excessive mumbling and to make many arguments cleaner, we will often use the Cohen Factorization Theorem stated below.

THEOREM. Let $A$ be a Banach algebra with an approximate identity bounded by 1 and let $X$ be a left Banach A-module. That is, $X$ is a Banach space and a left $A$-module, where $\|a x\| \leqslant\|a\|\|x\|$ for all $a \in A, x \in X$. Suppose the set of products $A X=\{a x \mid a \in A, x \in X\}$ is dense in $X$. Then, given $x \in X$ and $\varepsilon>0$ we can find $a \in X$ and $y \in X$ with $x=a y$, where $\|a\| \leqslant 1$ and $\|x-y\|<\varepsilon$.

Proof. See [9, p. 268].

The flip side of factorization is multiplication. Since many of the algebras we deal with have no unit, we are forced to consider multiplier algebras. We shall denote the multiplier algebra of a $C^{*}$-algebra, $A$, by $M(A)$. It is a standard result that a faithful, nondegenerate representation, $\pi$, of $A$ on a Hilbert space, $H$, extends to an isomorphism, $\bar{\pi}$, of $M(A)$ onto

$$
\{T \in \mathscr{B}(H) \mid T \pi(a) \text { and } \pi(a) T \in \pi(A) \text { for all } a \in A\}
$$

(see, for example, $[16,3.12 .3])$. We shall often use this fact without comment. We shall of ten consider the strict topology on $M(A)$, that is the topology induced by the seminorms $m \mapsto\|m a\|$ and $m \mapsto\|a m\|$ for $a \in A, m \in M(A)$. In general, $\bar{\phi}$ will 
denote the strictly continuous extension to $M(A)$ of a homomorphism $\phi$ defined on $A$; this extension exists for $\phi$ a nondegenerate representation, or a surjection, and in the following important situation. This lemma is well known: we include a proof only because we lack a reference.

1.1. Lemma. Let $\phi: B \rightarrow M(D)$ be a homomorphism of $C^{*}$-algebras and suppose there is an approximate identity $\left\{e_{i}\right\}$ for $B$ such that $\phi\left(e_{i}\right) \rightarrow 1$ strictly in $M(D)$. Then there is a unique strictly continuous extension $\bar{\phi}: M(B) \rightarrow M(D)$ defined by

$$
\begin{array}{ll}
\bar{\phi}(m) \cdot d=\phi\left(m b_{1}\right) c_{1} & \text { when } d=\phi\left(b_{1}\right) c_{1}, \\
d \cdot \bar{\phi}(m)=c_{2} \phi\left(b_{2} m\right) & \text { when } d=c_{2} \phi\left(b_{2}\right) .
\end{array}
$$

Proof. By the hypotheses, $D$ is a Banach $B$-module in which products are dense, and so every $d \in D$ factors as indicated. One easily checks that it is well defined and that $\|\bar{\phi}(m)\| \leqslant m$. It is also easily seen to be strictly continuous so that it is unique since $D$ is strictly dense in $M(D)$.

Now, if $A$ and $B$ are $C^{*}$-algebras, then $A \otimes B$ will denote their spatial tensor product, which is characterized by the property that $A \otimes B$ is represented faithfully on $H \otimes K$ whenever $A \subseteq \mathscr{B}(H)$ and $B \subseteq \mathscr{B}(K)$. We shall frequently use the following subalgebra of $M(A \otimes B)$ :

$$
\tilde{M}(A \otimes B)=\{m \in M(A \otimes B) \mid m(1 \otimes b),(1 \otimes b) m \in A \otimes B \text { for all } b \in B\} .
$$

Some intuition for this strange-looking object can be obtained from the special cases where $B$ has an identity, when $\tilde{M}(A \otimes B)=A \otimes B$; and where $B$ is commutative, when $\tilde{M}\left(A \otimes C_{0}(X)\right)=C_{b}(X, A)$. Roughly speaking, $\tilde{M}(A \otimes B)$ is somewhat larger than $A \otimes M(B)$. One of the most important properties of $\tilde{M}(A \otimes B)$ is that when you slice it by a function in $B^{*}$, you always end up in $A$ (see below for an explanation of this). When we come to defining unitary coactions, the following lemma concerning $\tilde{M}(A \otimes B)$ will be useful.

1.2. Lemma. Let $W$ be a unitary in $M(A \otimes B)$. Then the following are equivalent:

(1) $\operatorname{Ad} W: A \otimes 1 \rightarrow \tilde{M}(A \otimes B)$;

(2) $W(a \otimes 1) \in \tilde{M}(A \otimes B)$ for all $a \in A$;

(3) $(1 \otimes b) W(a \otimes 1) \in A \otimes B$ for all $a \in A, b \in B$.

Proof. Statements (2) and (3) are clearly equivalent. So, suppose (1) holds. Then for all $a \in A, b \in B$ :

$$
\begin{aligned}
(1 \otimes b) W(a \otimes 1) & =(1 \otimes b)[\operatorname{Ad} W(a \otimes 1)] W \\
& \in(1 \otimes b) \tilde{M}(A \otimes B) W \subseteq A \otimes B .
\end{aligned}
$$

So, (3) holds. Now, suppose (2) holds. Then factoring $a=a_{1} a_{2}^{*}$ in $A$ we get

$$
W(a \otimes 1) W^{*}(1 \otimes b)=\left[W\left(a_{1} \otimes 1\right)\right]\left[W\left(a_{2} \otimes 1\right)\right]^{*}(1 \otimes b) \in A \otimes B .
$$

But, trivially $(1 \otimes B) W(a \otimes 1) W^{*} \in A \otimes B$ and so (1) holds.

REMARK. Although, at first sight, it might seem conceivable that condition (3) always holds for unitaries $W$ in $M(A \otimes B)$, this is not the case. For example, 
consider $A=B=K(H)$, so that

$$
A \otimes B \cong K(H \otimes H) \quad \text { and } \quad M(A \otimes B) \cong \mathscr{B}(H \otimes H) .
$$

Define a unitary operator $W$ in $\mathscr{B}(H \otimes H)$ by $W(\xi \otimes \eta)=\eta \otimes \xi$. Then one easily calculates $(1 \otimes b) W(a \otimes 1)=1 \otimes b a$ which is not compact (for $H$ infinite-dimensional) and so is not in $A \otimes B \cong K(H \otimes H)$.

On the other hand, we have the following proposition.

1.3. Proposition. If $B$ is a commutative $C^{*}$-algebra and $W$ is a unitary in $M(A \otimes B)$, then the equivalent conditions of Lemma 1.2 hold.

Proof. Let $B=C_{0}(X)$ for $X$ locally compact. Then, by [1, Corollary 3.4], $M(A \otimes B)=C_{s}^{b}(X, M(A))$ where $C_{s}^{b}$ denotes strictly continuous, bounded functions. Let $W$ be a unitary in $M(A \otimes B)$ and let $a, b$ be in $A, B$ respectively. Then $a \otimes 1$ is a (constant) norm-continuous function into $A$ and so $W(a \otimes 1)$ is a norm-continuous bounded function into $A$. Since the function $b$ vanishes at $\infty$, $(1 \otimes b) W(a \otimes 1)$ is a norm-continuous function from $X$ into $A$ which vanishes at $\infty$. That is $(1 \otimes b) W(a \otimes 1) \in A \otimes B$ as required.

In $\S 4$ we will be considering ideals, $I$, in a $C^{*}$-algebra, $A$. Then, any multiplier $m$ of $A$ restricts to a multiplier $r(m)$ of $I$; note that the homomorphism $r$ is, in general, neither injective nor surjective. However, if $B$ is another $C^{*}$-algebra and we regard $I \otimes B$ as an ideal in $A \otimes B$, then we can view $\tilde{M}(I \otimes B)$ as a subalgebra of $\tilde{M}(A \otimes B)$ :

1.4. Lemma. The restriction homomorphism $r: M(A \otimes B) \rightarrow M(I \otimes B)$ is an isomorphism of $D=\{m \in \tilde{M}(A \otimes B) \mid x m, m x \in I \otimes B$ for all $x \in A \otimes B\}$ onto $\tilde{M}(I \otimes B)$.

Proof. If we let $1_{I}, 1_{A}$ denote the identities in $I^{* *}, A^{* *}$ respectively, then it is easy to see that for each $b \in B, r\left(1_{A} \otimes b\right)=1_{I} \otimes b$. Thus, for any $m \in D$,

$$
r(m)\left(1_{I} \otimes b\right)=r(m) r\left(1_{A} \otimes b\right)=r\left(m\left(1_{A} \otimes b\right)\right) .
$$

Now, $m\left(1_{A} \otimes b\right) \in A \otimes B$ and so $m\left(1_{A} \otimes b\right)=\lim _{j, k} m\left(e_{j} \otimes b f_{k}\right) \in I \otimes B$ by the definition of $D$, where $\left\{e_{j}\right\}$ and $\left\{f_{k}\right\}$ are approximate identities for $A$ and $B$, respectively. Thus,

$$
r(m)\left(1_{I} \otimes b\right)=r\left(m\left(1_{A} \otimes b\right)\right)=m\left(1_{A} \otimes b\right) \in I \otimes B,
$$

and so $r(m) \in \tilde{M}(I \otimes B)$.

If $r(m)=0$, then $m\left(1_{A} \otimes b\right)=0$ for all $b \in B$ and so $m=0$. On the other hand, if $y \in \tilde{M}(I \otimes B)$, then we can define a multiplier $i(y) \in M(A \otimes B)$ via $i(y) \cdot x=$ $y r(x)$ and $x \cdot i(y)=r(x) y$ for $x \in A \otimes B$. To see that this makes sense and, in fact, $i(y) \in D$, we observe that for $a \in A$ and $b \in B$

$$
\begin{aligned}
i(y)(a \otimes b) & =y r(a \otimes b)=y r\left(1_{A} \otimes b\right) r\left(a \otimes 1_{B}\right) \\
& =\left[y\left(1_{I} \otimes b\right)\right] r\left(a \otimes 1_{B}\right) \in(I \otimes B) M(I \otimes B)=I \otimes B .
\end{aligned}
$$

Thus, $i(y) \in D$ and we easily check that $r(i(y))=y$. 
Slice maps. If $A$ and $B$ are $C^{*}$-algebras and $f \in B^{*}$, then the linear map

$$
S_{f}\left(\sum_{i=1}^{n} a_{i} \otimes b_{i}\right)=\sum_{i=1}^{n} a_{i} f\left(b_{i}\right)
$$

extends to a bounded linear map of $A \otimes B$ into $A$ of norm $\|f\|$. Such maps are called slice maps [25]. The existence and the norm equality $\left\|S_{f}\right\|=\|f\|$ are obtained by viewing $A$ and $B$ on Hilbert spaces $H, K$ where $f$ becomes a vector functional on $\mathscr{B}(K)$. Then $A \otimes B$ is contained in the von Neumann algebra $A^{\prime \prime} \bar{\otimes} B^{\prime \prime}$ and $S_{f}$ is just the restriction of the corresponding weak-operator continuous slice maps $A^{\prime \prime} \bar{\otimes} B^{\prime \prime} \rightarrow A^{\prime \prime}$. The proof of the equality $\left\|S_{f}\right\|=\|f\|$ now follows from standard von Neumann algebra techniques.

1.5. Lemma. The restriction of $S_{f}$ to $M(A \otimes B) \subseteq A^{\prime \prime} \bar{\otimes} B^{\prime \prime}$ maps $M(A \otimes B)$ to $M(A)$ and $\tilde{M}(A \otimes B)$ to $A$. Moreover, $S_{f}$ is strictly continuous and if we factor $f=g_{1} \cdot b_{1}=b_{2} \cdot g_{2}$ for $b_{i} \in B, g_{i} \in B^{*}$, then

$$
S_{f}(m) \cdot a=S_{g_{1}}\left(m\left(a \otimes b_{1}\right)\right) \text { and } a \cdot S_{f}(m)=S_{g_{2}}\left(\left(a \otimes b_{2}\right) m\right)
$$

for all $a \in A$.

Proof. Using the Cauchy-Schwartz Theorem for positive functionals and the polar decomposition for arbitrary (continuous) functionals, one shows that $B \cdot B^{*}$ and $B^{*} \cdot B$ are each dense in $B^{*}$ and so every element $f \in B^{*}$ factors as above. As $f$ is weak-operator continuous on $B^{\prime \prime}$ these factorizations hold when we consider $f$ as a functional on $B^{\prime \prime}$. Using weak continuity, the equations

$$
S_{f}(x) \cdot a=S_{g_{1}}\left(x\left(a \otimes b_{1}\right)\right) \text { and } a \cdot S_{f}(x)=S_{g_{2}}\left(\left(a \otimes b_{2}\right) x\right)
$$

are easy to verify for all $x \in A^{\prime \prime} \bar{\otimes} B^{\prime \prime}$. Thus, for $m \in M(A \otimes B)$ we see that $S_{f}(m) \in M(A)$. Moreover, if $m \in \tilde{M}(A \otimes B)$, then the equation $S_{f}(m)=$ $S_{g_{1}}\left(m\left(1 \otimes b_{1}\right)\right)$ shows that $S_{f}(m) \in A$ as claimed. Now, if $m_{\alpha} \rightarrow m$ strictly, then

$$
S_{f}\left(m_{\alpha}\right) \cdot a=S_{g_{1}}\left(m_{\alpha}\left(a \otimes b_{1}\right)\right) \rightarrow S_{g_{1}}\left(m\left(a \otimes b_{1}\right)\right)=S_{f}(m) \cdot a .
$$

Combining this with a similar argument on the other side shows the strict continuity of $S_{f}$.

The regular representation of a locally compact group. If $G$ is a locally compact group, we shall write $\tau$ (respectively, $\sigma$ ) for the action of $G$ on $C_{0}(G)$ by left (respectively, right) multiplication; and $\lambda$ (respectively, $\rho$ ) for the left (respectively, right) regular representation of $G$ on $L^{2}(G)$. We shall identify the range of $\lambda$ : $C^{*}(G) \rightarrow \mathscr{B}\left(L^{2}(G)\right)$ with the reduced group $C^{*}$-algebra, $C_{r}^{*}(G)$, and denote the von Neumann algebras $\lambda(G)^{\prime \prime}, \rho(G)^{\prime \prime}$ by $L(G), R(G)$ respectively. There is a natural pairing between $C_{r}^{*}(G)$ and the algebra, $B_{r}(G)$, of continuous coefficient functions (for the representations of $C_{r}^{*}(G)$ ) defined by $\langle\lambda(g), f\rangle=\int_{G} g(t) f(t) d t$ for $g \in L^{1}(G)$ and $f \in B_{r}(G)$; this identifies the Fourier algebra, $A(G)$, with the predual of $L(G)$ [4]. Now, if $f$ is in $A(G)$, then $f$ is a coefficient function of the left regular representation: that is, there are $\psi, \phi \in L^{2}(G)$ so that

$$
\langle\lambda(g), f\rangle=\omega_{\psi \phi}(\lambda(g))=(\lambda(g) \psi \mid \phi) .
$$


In this case, one easily computes that $\bar{f}=\phi * \psi^{b}$, where $\psi^{b}(x)=\overline{\psi\left(x^{-1}\right)}$ : since we work on the left, we use Takesaki's original definition of $b[23]$, rather than that of [14]. In general, we shall use $\langle\cdot, \cdot\rangle$ to denote dual (bilinear) pairings and $(\cdot \mid \cdot)$ for the inner product on Hilbert spaces (conjugate-linear in the second variable).

Now, let Aut $A$ be the group of automorphisms of the $C^{*}$-algebra, $A$, and suppose $\alpha: G \rightarrow$ Aut $A$ is an action of $G$ on $A$ (always assumed to be strongly continuous). If $\pi$ is a representation of $A$ on a Hilbert space $H$, there is a natural representation Ind $\pi$ of the crossed product $A \times{ }_{\alpha} G$ on $L^{2}(G, H)$; in fact,

$$
(\text { Ind } \pi(\phi) \xi)(s)=\int_{G} \pi\left(\alpha_{s}^{-1}(\phi(t))\right) \xi\left(t^{-1} s\right) d t
$$

for $\phi \in C_{c}(G, A)$ and $\xi \in L^{2}(G, H)$. Of course, when $\pi$ is faithful, Ind $\pi$ is a faithful representation of the reduced crossed product $A \times_{\alpha, r} G[16,7.7 .5]$.

We shall move freely through the isomorphisms $H \otimes L^{2}(G) \cong L^{2}(G, H)$ and $L^{2}(G \times G) \cong L^{2}(G) \otimes L^{2}(G)$. If $W \in \mathscr{B}\left(L^{2}(G \times G)\right)$, then $W^{\sigma}$ will denote $W$ with the variables swapped: more precisely, $W^{\sigma}=\sum W \sum^{*}$ where $\left(\sum \xi\right)(s, t)=\xi(t, s)$ for $\xi \in L^{2}(G \times G), s, t \in G$. Finally, as a point of notation, $i$ will denote an identity mapping and 1 will denote the unit of a $C^{*}$-algebra.

Principal bundles. Let $E$ be a locally compact Hausdorff space on which $G$ acts freely (on the left). We will call $p: E \rightarrow E / G$ a (Cartan) principal $G$-bundle if the map $G \times E \rightarrow E \times E:(s, x) \mapsto(s x, x)$ is a proper map, in the sense that the preimage of each compact set is compact. By [15, Theorem 1.2.9] this implies that $E / G$ is a locally compact Hausdorff space and that $p: E \rightarrow E / G$ is a principal bundle in the sense of Cartan. If $G$ is a Lie group, then by [15, p. 315] this notion is equivalent to local triviality. In general, however, local triviality is a stronger condition and so we will take care to keep the notions distinct.

2. Coactions, crossed products, and exterior equivalence. For a locally compact group $G$, we denote by $W_{G}$ the unitary operator on $L^{2}(G \times G)$ defined by

$$
\left(W_{G} \xi\right)(s, t)=\xi\left(s, s^{-1} t\right) \quad \text { for } \xi \in L^{2}(G \times G) ; s, t \in G .
$$

Routine calculations show that for $\lambda(g) \in C_{r}^{*}(G)$, the operator $\delta_{G}(\lambda(g))=$ $W_{G}(\lambda(g) \otimes 1) W_{G}^{*}$ belongs to the algebra $\tilde{M}\left(C_{r}^{*}(G) \otimes C_{r}^{*}(G)\right)$. For example, if $f, g \in L^{1}(G)$, then $\delta_{G}(\lambda(g))(1 \otimes \lambda(f)) \xi=\rho * \xi$ for $\xi \in L^{2}(G \times G)$, where $\rho \in$ $L^{1}(G \times G)$ is given by $\rho(s, t)=g(s) f\left(s^{-1} t\right)$. Similarly, if $\left\{\lambda\left(e_{i}\right)\right\}$ is an approximate identity for $C_{r}^{*}(G)$, then $\delta_{G}\left(\lambda\left(e_{i}\right)\right) \rightarrow 1$ strictly in $M\left(C_{r}^{*}(G) \otimes C_{r}^{*}(G)\right)$. The necessary formulas are given in [13, p. 255]: take $A=\mathbf{C}, \rho=\mathrm{id}$, and note that our $W_{G}$ is $W^{*}$ in the notation of [13].

2.1. Definitron. A coaction of a locally compact group $G$ on a $C^{*}$-algebra $A$ is an injection $\delta$ of $A$ into $\tilde{M}\left(A \otimes C_{r}^{*}(G)\right)$ satisfying:

(a) there is an approximate identity $\left\{e_{i}\right\}$ for $A$ such that $\delta\left(e_{i}\right) \rightarrow 1$ strictly in $\tilde{M}\left(A \otimes C_{r}^{*}(G)\right)$,

(b) $(\delta \otimes i)^{-}(\delta(a))=\left(i \otimes \delta_{G}\right)^{-}(\delta(a))$ for all $a \in A$.

We say the coaction $\delta$ is nondegenerate if, in addition,

(c) for every nonzero $\phi \in A^{*}$ there exists $\psi \in C_{r}^{*}(G)^{*}$ such that $(\phi \otimes \psi)^{-} \circ \delta \neq 0$. 
2.2. RemarKs. (1) If $\left\{e_{i}\right\}$ is the approximate identity for $A$ described in (a), and $\left\{f_{k}\right\}$ is an approximate identity for $C_{r}^{*}(G)$, then $(\delta \otimes i)\left(e_{i} \otimes f_{k}\right) \rightarrow 1$ strictly in $M\left(A \otimes C_{r}^{*}(G) \otimes C_{r}^{*}(G)\right)$ and so $\delta \otimes i$ extends uniquely to a mapping of $M\left(A \otimes C_{r}^{*}(G)\right)$ into $M\left(A \otimes C_{r}^{*}(G) \otimes C_{r}^{*}(G)\right)$ by Lemma 1.1. Another application of Lemma 1.1 shows that $i \otimes \delta_{G}$ also extends, so condition (a) ensures that the coaction identity (b) makes sense.

(2) There are several equivalent formulations of condition (c), the definition of nondegeneracy [12, Theorem 5]. We shall find particularly useful the fact that $\delta$ is nondegenerate if and only if $A \otimes C_{r}^{*}(G)$ is the closed linear span of the set $\delta(A)\left(1 \otimes C_{r}^{*}(G)\right)$.

(3) It is not known whether every coaction is automatically nondegenerate, but this is the case when $G$ is amenable (see $[13 ; 12$, Proposition 6]). The proofs for discrete groups given in $[12,13]$ are unfortunately incorrect. One suspects that automatic nondegeneracy may be an amenable phenomenon.

(4) When the group $G$ is abelian, a coaction of $G$ is given by an action of the dual group $\hat{G}$. To see this, let $\mathscr{F}: L^{2}(G) \rightarrow L^{2}(\hat{G})$ denote the usual Fourier transform, so that $\mathscr{F} C_{r}^{*}(G) \mathscr{F}^{*}=C_{0}(\hat{G})$. Let $V \in U\left(L^{2}(\hat{G} \times \hat{G})\right)$ be defined by

$$
V \xi(\gamma, \chi)=\xi(\gamma \chi, \chi) \text { for } \xi \in L^{2}(\hat{G} \times \hat{G}), \gamma, \chi \in \hat{G} .
$$

Now, actions of $\hat{G}$ on $A$ can be viewed as injections $\pi$ of $A$ into $C_{b}(\hat{G}, A)=$ $\tilde{M}\left(A \otimes C_{0}(\hat{G})\right)$ as follows: given an action $\alpha$, define $\pi$ by $\pi(a)(\gamma)=\alpha_{\gamma}(a)$; the multiplicativity of $\alpha$ translates into the identity

$$
(\pi \otimes i)^{-}(\pi(a))=(1 \otimes V)(\pi(a) \otimes 1)\left(1 \otimes V^{*}\right)
$$

in $M\left(A \otimes C_{0}(\hat{G}) \otimes C_{0}(\hat{G})\right)$. It is then a matter of routine calculation to check that $\delta(a)=(1 \otimes \mathscr{F} *) \pi(a)(1 \otimes \mathscr{F})$ is a coaction of $G$ on $A$ and that the correspondence $\alpha \leftrightarrow \delta$ gives a bijection between coactions of $G$ on $A$ and (strongly continuous) actions of $\hat{G}$ on $A$. We observe that conditions (a) and (c) are automatic in this case: (a) by a compactness argument and (c) since abelian groups are amenable.

2.3. ExAmples. (1) The dual coaction. If $\alpha: G \rightarrow$ Aut $A$ is an action, and $\pi$ : $A \rightarrow \mathscr{B}(H)$ is faithful, then there is a faithful representation Ind $\pi$ of the reduced crossed product $A \times{ }_{\alpha r} G$ on $H \otimes L^{2}(G)$. In this case,

$$
((\operatorname{Ind} \pi) \otimes i)(\hat{\alpha}(x))=\left(1 \otimes W_{G}\right)(\operatorname{Ind} \pi(x) \otimes 1)\left(1 \otimes W_{G}^{*}\right)
$$

for $x \in A \times_{\alpha, r} G$ defines a nondegenerate coaction, $\hat{\alpha}$, of $G$ on $A \times_{\alpha, r} G$, called the dual coaction. This follows from the calculations in [13, pp. 255-257]. This is a special case of (6) below.

(2) $\delta_{G}$, the canonical coaction of $G$ on $C_{r}^{*}(G)$. If we realize $C_{r}^{*}(G)$ as the reduced crossed product of $G$ acting (trivially) on $\mathbf{C}$, then this is a special case of (1) above.

(3) Unitary coactions. Suppose we have a unitary $W \in M\left(A \otimes C_{r}^{*}(G)\right)$ which satisfies:

(a) $(W \otimes 1)(W \otimes 1)^{\sigma}=\left(1 \otimes W_{G}\right)(W \otimes 1)\left(1 \otimes W_{G}^{*}\right) ;$ 
(b) $W(a \otimes 1) \in \tilde{M}\left(A \otimes C_{r}^{*}(G)\right)$ for all $a \in A$.

Then condition (b) and Lemma 1.2 show that $\delta(a)=W(a \otimes 1) W^{*}$ is an injection of $A$ into $\tilde{M}\left(A \otimes C_{r}^{*}(G)\right)$, and condition (a) shows that $\delta$ is a coaction. The coaction $\delta$ will be nondegenerate if and only if condition (c) holds:

(c) $\left(1 \otimes C_{r}^{*}(G)\right) W(A \otimes 1)$ spans a dense subspace of $A \otimes C_{r}^{*}(G)$.

If conditions (a)-(c) hold we shall say that $\delta$ is a unitary coaction of $G$ on $A$, and that $W$ implements $\delta$. We do not know if condition (c) is necessary or if it is a consequence of the other conditions. Of course, when $G$ is abelian and $\delta$ corresponds to the action $\alpha: \hat{G} \rightarrow$ Aut $A$, then $\delta$ is unitary if and only if $\alpha$ is implemented by a strictly continuous homomorphism of $\hat{G}$ into $U(M(A))$. The next example is also a unitary coaction.

(4) The canonical (unitary) coaction of $G$ on $K\left(L^{2}(G)\right.$ ). For any locally compact group $G, W_{G}$ belongs to $M\left(K\left(L^{2}(G)\right) \otimes C_{r}^{*}(G)\right)$ and satisfies conditions (a)-(c) of (3). To see this recall that the crossed product $C_{0}(G) \times{ }_{\tau} G$ can be identified with $K\left(L^{2}(G)\right)$ via the representation $M \times \lambda$ on $L^{2}(G)$. In this case, $M \times \lambda$ is given by

$$
(M \times \lambda)(\phi) \xi(t)=\int \phi(t, r) \xi\left(r^{-1} t\right) d r \quad \text { for } \phi \in C_{c}(G \times G), \xi \in L^{2}(G) .
$$

To see that $W_{G}$ is a multiplier as indicated, one computes that

$$
W_{G}((M \times \lambda)(\phi) \otimes \lambda(g))=[(M \times \lambda) \otimes \lambda]\left(\psi_{1}\right)
$$

for $\phi \in C_{c}(G \times G), \quad g \in C_{c}(G)$, where $\psi_{1} \in C_{c}(G \times G \times G)$ is defined by $\psi_{1}(s, r, q)=\phi(s, r) g\left(s^{-1} q\right)$. Similarly, one shows that $((M \times \lambda)(\phi) \otimes \lambda(g)) W_{G}=$ $[(M \times \lambda) \otimes \lambda]\left(\psi_{2}\right)$, where $\psi_{2}$ is defined by $\psi_{2}(s, r, q)=\phi(s, r) \Delta\left(r s^{-1}\right) g\left(q r s^{-1}\right)$ and $\Delta$ is the modular function of $G$. Another calculation yields

$$
(1 \otimes \lambda(g)) W_{G}((M \times \lambda)(\phi) \otimes 1)=[(M \times \lambda) \otimes \lambda]\left(\psi_{3}\right),
$$

where $\psi_{3}(s, r, q)=\phi(s, r) \Delta\left(s^{-1}\right) g\left(q s^{-1}\right)$. It is easy to see that such $\psi_{3}$ 's span a dense subspace of $K\left(L^{2}(G)\right) \otimes C_{r}^{*}(G)$ and so conditions (b) and (c) of (3) are satisfied. Condition (a) is a routine computation, so that $\delta(T)=W_{G}(T \otimes 1) W_{G}^{*}$ defines a nondegenerate unitary coaction of $G$ on $K\left(L^{2}(G)\right)$. This example will be particularly important later. Of course, this example is another special case of example (1).

One should note that $W_{G}$ is not in $M\left(C_{r}^{*}(G) \otimes C_{r}^{*}(G)\right)$ (unless $G$ is the trivial group) and so $\delta_{G}$ is not a unitary coaction of $G$ on $C_{r}^{*}(G)$.

(5) Dual coaction on a reduced twisted crossed product. Let $G$ be a locally compact group with a closed normal subgroup, $N$. Let $\alpha: G \rightarrow$ Aut $A$ be an action of $G$ on $A$ and let $\mathscr{T}: N \rightarrow U(M(A))$ be a strictly continuous representation of $N$. As in [8], we define the triple $(G, A, \mathscr{T})$ to be a twisted covariant system if the following conditions are satisfied:

(a) $\alpha_{n}=\operatorname{Ad}(\mathscr{T}(n))$ for $n \in N$; and

(b) $\mathscr{T}\left(s n s^{-1}\right)=\alpha_{s}^{-}(\mathscr{T}(n))$ for $n \in N, s \in G$.

We say a representation $\pi \times U$ of $A \times{ }_{\alpha} G$ preserves $\mathscr{T}$ if $\bar{\pi}(\mathscr{T}(n))=U_{n}$ for all $n \in N$ and we define $C^{*}(G, A, \mathscr{T})$ to be $A \times{ }_{\alpha} G / I_{\mathscr{V}}$, where $I_{\mathscr{Y}}$ is the intersection of the kernels of all the representations which preserve $\mathscr{T}$. 
Now, let $\pi: A \rightarrow \mathscr{B}(H)$ be a faithful representation and let $C_{r}{ }^{*}(G, A, \mathscr{T})$ be the range of the representation $\operatorname{Ind}_{N}^{G} \pi$ of $C^{*}(G, A, \mathscr{T})$ : it can be shown using [8, Proposition 9, (iii)] that this is independent of the representation, $\pi$. In fact, if $G / N$ is amenable, then $C_{r}^{*}(G, A, \mathscr{T})$ is isomorphic to $C^{*}(G, A, \mathscr{T})$ by [8, Proposition 13]. Now the representation $\operatorname{Ind}_{N}^{G} \pi$ is given by $\pi \times \operatorname{Ind}_{N}^{G}(\bar{\pi} \circ \mathscr{T})$ acting on $A \times{ }_{\alpha} G$ : this latter representation preserves $\mathscr{T}$ and so indeed defines a representation of $C^{*}(G, A, \mathscr{T})$. The representation space of $\pi \times \operatorname{Ind}_{N}^{G}(\bar{\pi} \circ \mathscr{T})$ is

$H=\left\{\xi: G \rightarrow H_{\pi} \mid \xi\right.$ is Borel; $\xi(s n)=\pi\left(\mathscr{T}(n)^{-1}\right)(\xi(s))$ for $s \in G, n \in N$;

$$
\text { and } \left.\int_{G / N}\|\xi(s N)\|^{2} d(s N)<+\infty\right\}
$$

For $\phi \in C_{c}(G, A) \subseteq A \times_{\alpha} G$ and $\xi \in H$ the action is defined by

$$
\left[\left(\pi \times \operatorname{Ind}_{N}^{G}(\bar{\pi} \circ \mathscr{T})\right)(\phi)\right] \xi(s)=\int_{G} \pi\left(\alpha_{s}^{-1}(\phi(t))\right)\left(\xi\left(t^{-1} s\right)\right) d t .
$$

If we view $H \otimes L^{2}(G / N)$ as functions from $G \times G / N$ to $H_{\pi}$ which satisfy the appropriate condition on the first variable, then we can define a unitary operator $W$ on $H \otimes L^{2}(G / N)$ via

$$
(W \xi)(s, t N)=\xi\left(s, s^{-1} t N\right) .
$$

It is routine to check that $W$ satisfies the corepresentation condition:

$$
(W \otimes 1)(W \otimes 1)^{\sigma}=\left(1 \otimes W_{G / N}\right)(W \otimes 1)\left(1 \otimes W_{G / N}^{*}\right)
$$

on $H \otimes L^{2}(G / N) \otimes L^{2}(G / N)$. Moreover, a calculation shows that for $g \in$ $C_{c}(G / N) \subseteq C_{r}^{*}(G / N)$ and $\phi \in C_{c}(G, A) \subseteq A \times{ }_{\alpha} G$ we have $W\left(\left(\pi \times \operatorname{Ind}_{N}^{G}(\bar{\pi} \circ \mathscr{T})\right)(\phi) \otimes 1\right) W^{*}(1 \otimes \lambda(g))=\left[\left(\pi \times \operatorname{Ind}_{N}^{G}(\bar{\pi} \circ \mathscr{T})\right) \otimes \lambda\right](\psi)$, where $\psi \in C_{c}(G \times G / N, A)$ is defined by $\psi(r, s N)=\phi(r) g\left(r^{-1} s N\right)$. Thus, we can define a coaction of $G / N$ on $C_{r}^{*}(G, A, \mathscr{T})$ by

$$
\delta\left(\operatorname{Ind}_{N}^{G} \pi(b)\right)=W\left(\operatorname{Ind}_{N}^{G} \pi(b) \otimes 1\right) W^{*} \quad \text { for } b \in C^{*}(G, A, \mathscr{T}) .
$$

The corepresentation condition for $W$ shows that $\delta$ satisfies the coaction condition and the above calculation (together with a similar calculation on the left) shows that $\delta$ maps into $\tilde{M}\left(C_{r}^{*}(G, A, \mathscr{T}) \otimes C_{r}^{*}(G / N)\right)$. If we let $i_{G}, i_{A}$ denote the natural maps of $G, A$ into $M\left(C_{r}^{*}(G, A, \mathscr{T})\right)$, then we have

$$
\bar{\delta}\left(i_{A}(a)\right)=i_{A}(a) \otimes 1, \quad \bar{\delta}\left(i_{G}(s)\right)=i_{G}(s) \otimes \lambda(s N) .
$$

It easily follows, using the original definition [13], that $\delta$ is nondegenerate.

This example is in fact a special case of the following example, although the translation is a nontrivial exercise.

(6) $C^{*}$-algebraic bundles. Let $\pi: B \rightarrow G$ be a $C^{*}$-algebraic bundle over the locally compact group $G$ as defined by J. M. G. Fell in [5]. Let $d s$ denote left Haar measure on $G$ and let $A$ be the $C^{*}$-completion of the section algebra $L^{1}(\mathscr{B}, d s)$, where $\mathscr{B}=\langle B, \pi, G\rangle$. As each element $b$ of $B$ defines a multiplier of $A$, we see that the 
map $\delta: \quad B \rightarrow M\left(A \otimes C_{r}^{*}(G)\right)$ given by $\delta(b)=b \otimes \lambda(\pi(b))$ is a well-defined *representation of $B$ and so extends to a map $\delta: A \rightarrow M\left(A \otimes C_{r}^{*}(G)\right)$. In fact, if $a: G \rightarrow B$ is a continuous, compactly supported section, then

$$
\delta(a)=\int_{G} \delta(a(s)) \otimes \lambda(s) d s,
$$

where the integral is taken in the sense of strict convergence. As in [13, p. 255] one shows that $\delta(A) \subseteq \tilde{M}\left(A \otimes C_{r}^{*}(G)\right)$. If $\left\{f_{i}\right\}$ is an approximate unit for $L^{1}(\mathscr{B}, d s)$, then one can check that $\delta\left(f_{i}\right) \rightarrow 1$ strictly as in [5, Theorem 13.1]. It is also easy to check that $\delta$ is nondegenerate.

One should be aware that if $G$ is nondiscrete, then most coactions do not arise in this fashion. For example, if $G$ is abelian, a coaction corresponds to an action of the dual group $\hat{G}$, and in the case of a $C^{*}$-algebraic bundle this action is given by $\alpha_{\gamma}(b)=\gamma(\pi(b)) b$ for $b \in B$ and $\gamma \in \hat{G}$. This action extends to an action $\alpha$ of $\hat{G}$ on $A=C^{*}\left(L^{1}(\mathscr{B}, d s)\right)$. Now, if $A$ is abelian, then it follows from [22, Theorems 1, 2] that $\hat{A}$ is a so-called "characteristic" principal $\hat{G}$-bundle and the action of $\hat{G}$ on $A$ is just the transpose of the action of $\hat{G}$ on $\hat{A}$. Of course, it is trivial to find actions of abelian groups on spaces which are not principal bundle actions: for example $\mathbf{Z}$ acting on $S^{1}$ by an irrational rotation. Thus, the corresponding coaction of $\mathbf{T}$ on $C\left(S^{1}\right)$ does not arise from a $C^{*}$-algebraic bundle over $\mathbf{T}$.

Note that [22] and this article have a trivial intersection: groups acting on abelian algebras in a trivial way.

We now turn to the definition of the crossed product by a coaction. First we observe that if $B$ is a nondegenerate subalgebra of $\mathscr{B}(H)$, then there is a natural embedding of $M(A \otimes B)$ in $M(A \otimes K(H))$. To see this, suppose $A$ acts faithfully and nondegenerately on $H_{1}$, so that we can view $A \otimes B, M(A \otimes B)$, and $M(A \otimes K(H))$ as subalgebras of $\mathscr{B}\left(H_{1} \otimes H\right)$. If $\left\{e_{i}\right\}$ is an approximate identity for $B$, then $e_{i} \rightarrow 1$ strongly on $H$, so $e_{i} k \rightarrow k$ in norm for every $k \in K(H)$. Thus for $m \in M(A \otimes B)$ we have

$$
m(a \otimes k)=\lim _{i} m\left(a \otimes e_{i} k\right)=\lim _{i} m\left(a \otimes e_{i}\right)(1 \otimes k)
$$

which clearly belongs to $A \otimes K$. Thus, in particular, we can regard $M\left(A \otimes C_{r}^{*}(G)\right)$ as a subalgebra of $M\left(A \otimes K\left(L^{2}(G)\right)\right)$.

2.4. Definition. Let $\delta$ be a coaction of a locally compact group $G$ on a $C^{*}$-algebra $A$. The crossed product $A \times_{\delta} G$ of $A$ by $\delta$ is the $C^{*}$-subalgebra of $M\left(A \otimes K\left(L^{2}(G)\right)\right)$ generated by the set $\left\{\delta(a)\left(1 \otimes M_{f}\right) \mid a \in A, f \in C_{0}(G)\right\}$.

We observe that for $f \in A(G), M_{f}=S_{f}\left(W_{G}\right)$-an important fact!

2.5. Lemma. Let $\delta$ be a coaction of $G$ on $A$. Then $a \mapsto \delta(a)$ and $f \mapsto 1 \otimes M_{f}$ embed $A$ and $C_{0}(G)$ in $M\left(A \times{ }_{\delta} G\right)$. Moreover, $A \times{ }_{\delta} G=\delta(A)\left(A \times{ }_{\delta} G\right)$ and $A \times{ }_{\delta} G=$ $\left(1 \otimes M_{C_{0}(G)}\right)\left(A \times_{\delta} G\right)$. Then

Proof. Let $\delta\left(b_{1}\right)\left(1 \otimes M_{f_{1}}\right)$ and $\left(1 \otimes M_{f_{2}}\right) \delta\left(b_{2}\right)$ be typical generators of $A \times{ }_{\delta} G$.

$$
\delta(a)\left[\delta\left(b_{1}\right)\left(1 \otimes M_{f_{1}}\right)\right]=\delta\left(a b_{1}\right)\left(1 \otimes M_{f_{1}}\right) \in A \times_{\delta} G
$$


and

$$
\delta(a)\left[\left(1 \otimes M_{f_{2}}\right) \delta\left(b_{2}\right)\right]=\left[\delta(a)\left(1 \otimes M_{g_{1}}\right)\right]\left[\left(1 \otimes M_{g_{2}}\right) \delta\left(b_{2}\right)\right] \in A \times_{\delta} G,
$$

where we have factored $f_{2}=g_{1} g_{2}$. Thus, $\delta(a) \in M\left(A \times{ }_{\delta} G\right)$ and similarly, $1 \otimes M_{f}$ $\in M\left(A \times_{\delta} G\right)$.

To see the factorization statements, we show that any element $\left(1 \otimes M_{f}\right) \delta(a)$ can be approximated by a finite sum $\sum_{i} \delta\left(b_{i}\right)\left(1 \otimes M_{g_{i}}\right)$ and so any element of $A \times_{\delta} G$ can be approximated by a finite sum $\sum_{j} \delta\left(a_{j}\right)\left(1 \otimes M_{f_{j}}\right) x_{j}$, where $x_{j} \in A \times_{\delta} G$. Letting $\left\{e_{k}\right\}$ be an approximate identity for $A$ we see that any element of $A \times{ }_{\delta} G$ is approximately $\delta\left(e_{k}\right) \cdot y$ for some $k$ and some $y \in A \times{ }_{\delta} G$. An application of Cohen's theorem will then complete the proof. A similar argument works for the second factorization statement. Now

$$
\begin{aligned}
\left(1 \otimes M_{f}\right) \delta(a) & =S_{f}\left(1 \otimes W_{G}\right) \delta(a)=S_{f}\left(\left(1 \otimes W_{G}\right)(\delta(a) \otimes 1)\right) \\
& =S_{f}\left(\left(1 \otimes W_{G}\right)(\delta(a) \otimes 1)\left(1 \otimes W_{G}^{*}\right)\left(1 \otimes W_{G}\right)\right) \\
& =S_{f}\left(\left(i \otimes \delta_{G}\right)^{-}(\delta(a))\left(1 \otimes W_{G}\right)\right) \\
& =S_{f}\left((\delta \otimes i)^{-}(\delta(a))\left(1 \otimes W_{G}\right)\right) \\
& =S_{x \cdot g}\left((\delta \otimes i)^{-}(\delta(a))\left(1 \otimes W_{G}\right)\right) \\
& =S_{g}\left((\delta \otimes i)((1 \otimes x) \delta(a))\left(1 \otimes W_{G}\right)\right)
\end{aligned}
$$

which is approximately

$$
\begin{aligned}
S_{g}(( & \left.\delta \otimes i)\left(\sum_{i} b_{i} \otimes x_{i}\right)\left(1 \otimes W_{G}\right)\right) \\
& =\sum_{i} \delta\left(b_{i}\right) S_{g}\left(\left(1 \otimes x_{i}\right)\left(1 \otimes W_{G}\right)\right)=\sum_{i} \delta\left(b_{i}\right) S_{x_{i} \cdot g}\left(1 \otimes W_{G}\right) \\
& =\sum_{i} \delta\left(b_{i}\right)\left(1 \otimes M_{g_{i}}\right), \quad \text { where } g_{i}=x_{i} \cdot g .
\end{aligned}
$$

2.6. Remarks. (1) Of course, by taking adjoints we get similar factorizations of $A \times{ }_{\delta} G$ on the right. These factorizations will be very important in $\S \S 3$ and 4 .

(2) When $G$ is abelian and $\delta$ corresponds to the automorphism group $\alpha: \hat{G} \rightarrow$ Aut $A$, then conjugating by $1 \otimes \mathscr{F}$ carries $\delta(a)$ into the function $\gamma \mapsto \alpha_{\gamma}(a)$ in $C_{b}(\hat{G}, A)$ and $1 \otimes M_{f}$ into $1 \otimes \lambda(\hat{f})$. These operators in $M\left(A \otimes K\left(L^{2}(\hat{G})\right)\right)$ generate the crossed product $A \times{ }_{\alpha} \hat{G}$, so our definition agrees with the usual one in this case.

(3) If $\phi: A \rightarrow B$ is an isomorphism such that $\phi \otimes i$ intertwines two coactions $\delta, \varepsilon$ of $G$ on $A, B$ respectively, then it is not too hard to see that $\phi \otimes i$ also gives an isomorphism of $A \times_{\delta} G$ onto $B \times{ }_{\varepsilon} G$. In particular, when $\pi: A \rightarrow \mathscr{B}(H)$ is a faithful nondegenerate representation, the crossed product $A \times{ }_{\delta} G$ is isomorphic to the $C^{*}$-subalgebra of $\mathscr{B}\left(H \otimes L^{2}(G)\right)$ generated by

$$
\left\{(\pi \otimes i)^{-}(\delta(a))\left(1 \otimes M_{f}\right) \mid a \in A, f \in C_{0}(G)\right\} .
$$

Thus, our definition of crossed product coincides with the spatial definition of Katayama [12]. Of course, it also follows that the spatially defined crossed product is independent of the chosen representation of $A$. 
We will now discuss exterior equivalence of coactions and show that exterior equivalent coactions give rise to isomorphic crossed products. As an immediate special case we deduce Quigg's result [19] on crossed products by unitary coactions. In $\S 3$ we will show how this isomorphism behaves with respect to representations of the crossed products. In $\S 5$ we will use these results to show that exterior equivalence of locally unitary coactions corresponds to isomorphism of the associated principal bundles.

2.7. Definition. Let $\delta$ be a coaction of $G$ on $A$ and let $u$ be a unitary in $M\left(A \otimes C_{r}^{*}(G)\right)$ satisfying:

(1) $\left(i \otimes \delta_{G}\right)^{-}(u)=(u \otimes 1)\left[(\delta \otimes i)^{-}(u)\right]$, and

(2) $u \delta(a) u^{*} \in \tilde{M}\left(A \otimes C_{r}^{*}(G)\right)$ for all $a \in A$.

Then, $\gamma=\operatorname{Ad} u \circ \delta$ is a coaction of $G$ on $A, u$ is called a $\delta$-one cocycle, and we say that $\delta$ and $\gamma$ are exterior equivalent. One easily sees that if $u$ is a $\delta$-one cocycle, then $u^{*}$ is a $\gamma$-one cocycle for $\gamma=\operatorname{Ad} u \circ \delta$, so that the definition is indeed symmetric. If $u$ also satisfies condition (3):

(3) $\left(1 \otimes C_{r}^{*}(G)\right) u \delta(A)$ is dense in $A \otimes C_{r}^{*}(G)$,

then $\gamma$ is a nondegenerate coaction by an application of [12, Theorem 5]. We do not know whether a coaction which is exterior equivalent to a nondegenerate coaction is necessarily nondegenerate, but we suspect that this is true.

2.8. REMARKS. If $\gamma=\operatorname{Ad} u \circ \delta$ is exterior equivalent to the trivial coaction $\delta(a)=a \otimes 1$, then $(\delta \otimes i)^{-}(u)=(u \otimes 1)^{\sigma}$ and so condition (1) becomes

$$
\left(1 \otimes W_{G}\right)(u \otimes 1)\left(1 \otimes W_{G}^{*}\right)=(u \otimes 1)(u \otimes 1)^{\sigma} .
$$

This is precisely condition (a) in the definition of a unitary coaction. By Lemma 1.2, condition (2) above then becomes condition (b) in the definition of a unitary coaction. In this setting, condition (3) above is identical to condition (c) of the previous definition. Thus, a coaction $\gamma$ is a unitary coaction if and only if it is nondegenerate and exterior equivalent to the trivial coaction.

2.9. ThEOREM. Let $\delta$ be a coaction of $G$ on $A$ (not necessarily nondegenerate) and let $u$ be a $\delta$-one cocycle so that $\gamma=\operatorname{Ad} u \circ \delta$ is a coaction of $G$ on $A$. Considering $u$ as an element of $M\left(A \otimes K\left(L^{2}(G)\right)\right)$ we have that $\operatorname{Ad} u: A \times{ }_{\delta} G \rightarrow A \times{ }_{\gamma} G$ is an isomorphism.

Proof. By symmetry, it suffices to see that $\operatorname{Ad} u\left(\delta(a)\left(1 \otimes M_{f}\right)\right) \in A \times_{\delta} G$ for $a \in A$ and $f \in A(G)$. To this end, we compute:

$$
\begin{aligned}
\operatorname{Ad} u\left(\delta(a)\left(1 \otimes M_{f}\right)\right) & =u \delta(a) u^{*} u\left(1 \otimes M_{f}\right) u^{*}=\gamma(a) u S_{f}\left(1 \otimes W_{G}\right) u^{*} \\
& =\gamma(a) S_{f}\left((u \otimes 1)\left(1 \otimes W_{G}\right)\left(u^{*} \otimes 1\right)\right) \\
& =\gamma(a) S_{f}\left((u \otimes 1)\left(1 \otimes W_{G}\right)\left(u^{*} \otimes 1\right)\left(1 \otimes W_{G}^{*}\right)\left(1 \otimes W_{G}\right)\right) \\
& =\gamma(a) S_{f}\left((u \otimes 1)\left(i \otimes \delta_{G}\right)^{-}\left(u^{*} \otimes 1\right)\left(1 \otimes W_{G}\right)\right) \\
& =\gamma(a) S_{f}\left((u \otimes 1)\left(u^{*} \otimes 1\right)(\gamma \otimes i)^{-}\left(u^{*}\right)\left(1 \otimes W_{G}\right)\right) \\
& =S_{f}\left((\gamma \otimes i)^{-}\left((a \otimes 1) u^{*}\right)\left(1 \otimes W_{G}\right)\right) .
\end{aligned}
$$


Now, we factor $f=g \cdot x$, where $g \in A(G)$ and $x \in C_{r}^{*}(G)$, so that we get $S_{g}\left((1 \otimes x)(\gamma \otimes i)^{-}\left((a \otimes 1) u^{*}\right)\left(1 \otimes W_{G}\right)\right)=S_{g}\left((\gamma \otimes i)\left((a \otimes x) u^{*}\right)\left(1 \otimes W_{G}\right)\right)$. As $(a \otimes x) u^{*} \in A \otimes C_{r}^{*}(G)$, this can be approximated by a finite sum $\sum b_{i} \otimes x_{i}$ with $b_{i} \in A$ and $x_{i} \in C_{r}^{*}(G)$. So, $\operatorname{Ad} u\left(\delta(a)\left(1 \otimes M_{f}\right)\right)$ is approximately equal to

$$
\begin{aligned}
S_{g}\left((\gamma \otimes i)\left(\sum b_{i} \otimes x_{i}\right)\left(1 \otimes W_{G}\right)\right) & =\sum \gamma\left(b_{i}\right)\left[1 \otimes S_{g}\left(\left(1 \otimes x_{i}\right) W_{G}\right)\right] \\
& =\sum \gamma\left(b_{i}\right)\left[1 \otimes S_{x_{i} \cdot g}\left(W_{G}\right)\right] \\
& =\sum \gamma\left(b_{i}\right)\left(1 \otimes M_{x_{i} \cdot g}\right)
\end{aligned}
$$

which is in $A \times{ }_{\gamma} G$.

2.10. Corollary. Let $\gamma(a)=W(a \otimes 1) W^{*}$ be a (not necessarily nondegenerate) unitary coaction of $G$ on $A$. Then, $\operatorname{Ad} W^{*}: A \times{ }_{\gamma} G \rightarrow A \otimes C_{0}(G)$ is an isomorphism.

3. Representations of crossed products. Let $\delta$ be a coaction of a locally compact group $G$ on a $C^{*}$-algebra $A$. Given a nondegenerate representation $\nu$ of the crossed product $A \times{ }_{\delta} G$ on $H$, we can define representations $\pi$ of $A$ and $\mu$ of $C_{0}(G)$ by composing $\bar{\nu}$ with the embeddings $\delta$ and $1 \otimes M$ of $A$ and $C_{0}(G)$ as subalgebras of $M\left(A \times{ }_{\delta} G\right)$. We want to find conditions on a pair of representations $(\pi, \mu)$ which are satisfied when they come from a representation $\nu$ of $A \times{ }_{\delta} G$, and, conversely, which will ensure that the map

$$
\delta(a)\left(1 \otimes M_{f}\right) \mapsto \pi(a) \mu(f)
$$

is well defined and extends to a representation of $A \times{ }_{\delta} G$. This will give an analogue for coactions of the usual notion of a covariant pair of representations of a $C^{*}$-dynamical system $(A, G, \alpha)$. We shall depend heavily, now and in later sections, on the following result of Nakagami and Takesaki [14, Theorem A.1(b)] on the representation theory of the Fourier algebra $A(G)$.

3.1. THEOREM. Let $G$ be a locally compact group. There is a bijection between the nondegenerate representations $\mu$ of $A(G)$ on a Hilbert space $H$ and unitaries $W \in$ $\mathscr{B}(H) \bar{\otimes} L(G)$ satisfying

$$
(W \otimes 1)(W \otimes 1)^{\sigma}=\left(1 \otimes W_{G}\right)(W \otimes 1)\left(1 \otimes W_{G}^{*}\right) ;
$$

this bijection is determined by the relation $\mu(f)=S_{f}(W)$ for $f \in A(G)$.

3.2. RemarKs. (1) If $W$ satisfies (*), then by an easy calculation

$$
(W \otimes 1)(W \otimes 1)^{\sigma}=(W \otimes 1)^{\sigma}(W \otimes 1) .
$$

Using this and taking adjoints of the equation $(*)$ we see that $W^{*}$ also satisfies this equation.

(2) We shall refer to a unitary $W \in \mathscr{B}(H) \bar{\otimes} L(G)$ satisfying (*) as a corepresentation of $G$ on $H$. When $G$ is abelian, of course, these are the Fourier transforms of the unitary representations of $\hat{G}$. We observe that the condition $(*)$ is formally the same as that which occurred in the definition of unitary coaction: in fact, if $W \in M\left(A \otimes C_{r}^{*}(G)\right)$ satisfies (a) of Example 2.3(3) and $\pi$ is a nondegenerate representation of $A$ on $H$, then $(\pi \otimes i)^{-}(W)$ is a corepresentation of $G$ on $H$. 
(3) It is easy to check that $W=W_{G}$ commutes with each $1 \otimes \rho(s)$ and hence belongs to $\mathscr{B}\left(L^{2}(G)\right) \bar{\otimes} L(G)$. Since $W_{G}$ satisfies $(*)$ and $M_{f}=S_{f}\left(W_{G}\right)$, we have that $M$ is the nondegenerate representation of $A(G)$ on $L^{2}(G)$ corresponding to $W_{G}$.

Returning to our problem of what is a covariant pair of representations for a coaction $\delta$, it seems that the appropriate conditions are more easily expressible in terms of the operator $W$ than in terms of the representation $\mu$ of $A(G)$. It turns out that if $\nu$ is a representation of $A \times{ }_{\delta} G$ and we let $\mu=\bar{\nu} \circ(1 \otimes M)$, then the corresponding $W$ is given by $(\nu \otimes i)^{-}\left(1 \otimes W_{G}\right)$ : our next lemma shows that this makes sense and will also be crucial later on.

3.3. Lemma. Suppose that $A$ acts nondegenerately and faithfully on $H$, so that $A \times{ }_{\delta} G$ acts faithfully and nondegenerately on $H \otimes L^{2}(G)$. Then

$$
1 \otimes W_{G} \in M\left(\left(A \times_{\delta} G\right) \otimes C_{r}^{*}(G)\right) \subseteq \mathscr{B}\left(H \otimes L^{2}(G) \otimes L^{2}(G)\right) .
$$

Proof. Let $x \otimes \lambda(g) \in\left(A \times_{\delta} G\right) \otimes C_{r}^{*}(G)$ and let $x=\left(1 \otimes M_{f}\right) y$ for $f \in$ $C_{0}(G), y \in A \times{ }_{\delta} G$ by Lemma 2.5. Then,

$$
\begin{aligned}
\left(1 \otimes W_{G}\right)(x \otimes \lambda(g)) & =\left(1 \otimes W_{G}\right)\left(1 \otimes M_{f} \otimes \lambda(g)\right)(y \otimes 1) \\
& =1 \otimes\left[W_{G}\left(M_{f} \otimes \lambda(g)\right)\right](y \otimes 1) .
\end{aligned}
$$

Now, for $g \in L^{1}(G)$ we see that $W_{G}\left(M_{f} \otimes \lambda(g)\right)$ is multiplication by the operatorvalued function $s \mapsto f(s) \lambda(s \cdot g)$, where $s \cdot g(r)=g\left(s^{-1} r\right)$. This belongs to $C_{0}\left(G, C_{r}^{*}(G)\right)=C_{0}(G) \otimes C_{r}^{*}(G)$ and so

$$
\begin{aligned}
\left(1 \otimes W_{G}\right)(x \otimes \lambda(g)) & \in\left(1 \otimes C_{0}(G) \otimes C_{r}{ }^{*}(G)\right)\left(\left(A \times_{\delta} G\right) \otimes 1\right) \\
& \subseteq\left(A \times_{\delta} G\right) \otimes C_{r}{ }^{*}(G)
\end{aligned}
$$

as required. A similar calculation shows that $1 \otimes W_{G}$ is also a right multiplier.

3.4. Proposition. Let $\delta$ be a coaction and let $\nu$ be a nondegenerate representation of $A \times{ }_{\delta} G$ on $H$. Define $\pi=\bar{\nu} \circ \delta, \mu=\bar{\nu} \circ(1 \otimes M)$, and $W=(\nu \otimes i)^{-}\left(1 \otimes W_{G}\right)$. Then $\pi$ and $\mu$ are nondegenerate representations of $A$ and $A(G)$ on $H$. Moreover, $W$ is the unitary in $\mathscr{B}(H) \bar{\otimes} L(G)$ which corresponds to $\mu$ and we have the covariance condition

$$
(\pi \otimes i)^{-}(\delta(a))=W(\pi(a) \otimes 1) W^{*} \quad \text { for } a \in A
$$

Proof. By the factorization results of Lemma 2.5 we see that $\pi$ and $\mu$ are nondegenerate whenever $\nu$ is nondegenerate. We have already observed in Remark 3.2(3) that $W_{G}$ satisfies condition (*) of Theorem 3.1 and hence $W$ also satisfies that condition. To see that $W$ corresponds to $\mu$, let $f \in A(G)$; then

$$
\mu(f)=\bar{\nu}\left(1 \otimes M_{f}\right)=\bar{\nu}\left(S_{f}\left(1 \otimes W_{G}\right)\right)=S_{f}\left((\nu \otimes i)^{-}\left(1 \otimes W_{G}\right)\right)=S_{f}(W)
$$

as required. 
Finally, to see the covariance condition, let $a \in A$; then

$$
\begin{aligned}
W(\pi(a) \otimes 1) W^{*} & =(\nu \otimes i)^{-}\left(1 \otimes W_{G}\right)(\bar{\nu}(\delta(a)) \otimes 1)(\nu \otimes i)^{-}\left(1 \otimes W_{G}^{*}\right) \\
& =(\nu \otimes i)^{-}\left(\left(1 \otimes W_{G}\right)(\delta(a) \otimes 1)\left(1 \otimes W_{G}^{*}\right)\right) \\
& =(\nu \otimes i)^{-}\left(\left(i \otimes \delta_{G}\right)^{-}(\delta(a))\right) \\
& =(\nu \otimes i)^{-}\left((\delta \otimes i)^{-}(\delta(a))\right) \\
& =(\nu \circ \delta \otimes i)^{-}(\delta(a))=(\pi \otimes i)^{-}(\delta(a)) .
\end{aligned}
$$

3.5. Definition. A pair $(\pi, W)$ consisting of a nondegenerate representation $\pi$ of $A$ on $H$, and a unitary $W \in \mathscr{B}(H) \bar{\otimes} L(G)$ which corresponds under Theorem 3.1 to a nondegenerate representation $\mu$ of $A(G)$ on $H$ will be called a covariant pair of representations of the system $(A, G, \delta)$ if for all $a \in A$

$$
(\pi \otimes i)^{-}(\delta(a))=W(\pi(a) \otimes 1) W^{*} .
$$

3.6. Lemma. Let $W \in \mathscr{B}(H) \bar{\otimes} L(G)$ be a corepresentation of $G$ on $H$. Then, for $f \in A(G)$, the operator $W^{*}\left(1 \otimes M_{f}\right) W$ is multiplication by the norm-continuous operator-valued function $s \rightarrow \mu\left(s^{-1} \cdot f\right)$, where $\mu$ is the corresponding representation of $A(G)$ and $s^{-1} \cdot f(t)=f(s t)$ for $s, t \in G$.

Proof. First we calculate:

$$
\begin{aligned}
W^{*}(1 \otimes & \left.M_{f}\right) W=W^{*} S_{f}\left(1 \otimes W_{G}\right) W=S_{f}\left(\left(W^{*} \otimes 1\right)\left(1 \otimes W_{G}\right)(W \otimes 1)\right) \\
& =S_{f}\left(\left(W^{*} \otimes 1\right)\left(1 \otimes W_{G}\right)(W \otimes 1)\left(1 \otimes W_{G}^{*}\right)\left(1 \otimes W_{G}\right)\right) \\
& =S_{f}\left(\left(W^{*} \otimes 1\right)(W \otimes 1)(W \otimes 1)^{\sigma}\left(1 \otimes W_{G}\right)\right)=S_{f}\left((W \otimes 1)^{\sigma}\left(1 \otimes W_{G}\right)\right) .
\end{aligned}
$$

Now, $(W \otimes 1)$ is just multiplication by the constant $\mathscr{B}(H) \bar{\otimes} L(G)$-valued function $s \mapsto W$ (for $s$ in the third variable), and so $(W \otimes 1)^{\circ}$ is just multiplication by the constant $\mathscr{B}(H) \bar{\otimes} L(G)$-valued function $s \mapsto W$ (for $s$ in the second variable). On the other hand, $\left(1 \otimes W_{G}\right)$ is just multiplication by the $\mathscr{B}(H) \bar{\otimes} L(G)$-valued function $s \mapsto(1 \otimes \lambda(s))$ for $s$ in the second variable. Thus $(W \otimes 1)^{\circ}\left(1 \otimes W_{G}\right)$ is multiplication by the $\mathscr{B}(H) \bar{\otimes} L(G)$-valued function $s \mapsto W(1 \otimes \lambda(s))$ for $s$ in the second variable. As $S_{f}$ indicates the slice map in the third variable, we have that $S_{f}\left((W \otimes 1)^{\sigma}\left(1 \otimes W_{G}\right)\right)$ is just multiplication by the $\mathscr{B}(H)$-valued function

$$
s \mapsto S_{f}(W(1 \otimes \lambda(s)))=S_{s^{-1} \cdot f}(W)=\mu\left(s^{-1} \cdot f\right) .
$$

The norm-continuity of translation in $A(G)$ implies $s \mapsto \mu\left(s^{-1} \cdot f\right)$ is norm-continuous from $G$ to $\mathscr{B}(H)$.

3.7. THEOREM. Let $\delta$ be a coaction of a locally compact group $G$ on a $C^{*}$-algebra A. The map $\nu \mapsto\left(\bar{\nu} \circ \delta,(\nu \otimes i)^{-}\left(1 \otimes W_{G}\right)\right)$ is a bijection between the nondegenerate representations of $A \times{ }_{\delta} G$ on $H$ and the covariant pairs of representations of $(A, G, \delta)$ on $H$.

Proof. In view of Proposition 3.4, it will be enough to show that for each covariant pair $(\pi, W)$ there is a nondegenerate representation $\nu$ of $A \times{ }_{\delta} G$ with $\pi=\bar{\nu} \circ \delta$ and $W=(\nu \otimes i)^{-}\left(1 \otimes W_{G}\right)$. So, let $(\pi, W)$ be a covariant pair of representations on $H$ and let $\mu$ be the corresponding nondegenerate representation of 
$A(G)$ on $H$. Consider the representation

$$
\tau=\operatorname{Ad} W^{*} \circ(\pi \otimes i)^{-}
$$

of $M\left(A \otimes K\left(L^{2}(G)\right)\right)$ on $H \otimes L^{2}(G)$. Restricting this representation to $A \times{ }_{\delta} G$, we have

$$
\begin{aligned}
\tau\left(\delta(a)\left(1 \otimes M_{f}\right)\right) & =W^{*}(\pi \otimes i)^{-}(\delta(a)) W W^{*}\left(1 \otimes M_{f}\right) W \\
& =(\pi(a) \otimes 1) W^{*}\left(1 \otimes M_{f}\right) W .
\end{aligned}
$$

By Lemma 3.6 this is just multiplication by the norm-continuous function $s \mapsto$ $\pi(a) \mu\left(s^{-1} \cdot f\right)$. Thus, $\tau$ maps $A \times_{\delta} G$ into $\left.C_{b}(G, B)\right)$, where $B$ is the $C^{*}$-algebra on $H$ generated by elements of the form $\pi(a) \mu(f)$. Thus, if we follow $\tau$ by evaluation at $e \in G$ we get a representation $\nu$ of $A \times{ }_{\delta} G$ on $H$, where

$$
\nu\left(\delta(a)\left(1 \otimes M_{f}\right)\right)=\pi(a) \mu(f) .
$$

Since $\pi$ and $\mu$ are nondegenerate, so is $\nu$. Clearly, $\bar{\nu} \circ \delta=\pi$ and $\bar{\nu} \circ(1 \otimes M)=\mu$. By Proposition 3.4, $(\nu \otimes i)^{-}\left(1 \otimes W_{G}\right)$ is the corepresentation corresponding to $\mu$ and so $W=(\nu \otimes i)^{-}\left(1 \otimes W_{G}\right)$ by Theorem 3.1 .

3.8. Notation. We will write $\pi \times W$ for the representation of $A \times{ }_{\delta} G$ corresponding to the covariant pair $(\pi, W)$ for the system $(A, G, \delta)$. We take time to record the equation:

$$
\pi \times W=\operatorname{Eval}_{e} \circ \operatorname{Ad} W^{*} \circ(\pi \otimes i)^{-},
$$

where $\mathrm{Eval}_{e}$ is self-explanatory.

3.9. Proposition. Let $\delta$ be a coaction of $G$ on $A$ and let $u$ be $a$-one cocycle so that $\gamma=\operatorname{Ad} u \circ \delta$ is also a coaction of $G$ on $A$. Let $\Phi=\operatorname{Ad} u: A \times{ }_{\delta} G \rightarrow A \times{ }_{\gamma} G$ be the isomorphism of Theorem 2.9. If $\pi \times W$ is a representation of $A \times{ }_{\gamma} G$, then

$$
\Phi^{*}(\pi \times W)=(\pi \times W) \circ \Phi=\pi \times\left((\pi \otimes i)^{-}\left(u^{*}\right) W\right)
$$

is the corresponding representation of $A \times_{\delta} G$.

Proof. By Theorem 3.7, $\Phi^{*}(\pi \times W)=\rho \times V$ for some $\delta$-covariant pair $(\rho, V)$. But, for $a \in A$

$$
\rho(a)=(\rho \times V)^{-}(\delta(a))=(\pi \times W)^{-}\left(u \delta(a) u^{*}\right)=(\pi \times W)^{-}(\gamma(a))=\pi(a),
$$
so we have $\Phi^{*}(\pi \times W)=\pi \times V$. Now, Theorem 3.7 also gives us the equation

$$
V=((\pi \times V) \otimes i)^{-}\left(1 \otimes W_{G}\right) \text {. }
$$

So, we compute

$$
\begin{aligned}
V & =((\pi \times V) \otimes i)^{-}\left(1 \otimes W_{G}\right)=([(\pi \times W) \circ \mathrm{Ad} u] \otimes i)^{-}\left(1 \otimes W_{G}\right) \\
& =((\pi \times W) \otimes i)^{-}\left((u \otimes 1)\left(1 \otimes W_{G}\right)\left(u^{*} \otimes 1\right)\right) \\
& =((\pi \times W) \otimes i)^{-}\left((u \otimes 1)\left(i \otimes \delta_{G}\right)\left(u^{*}\right)\left(1 \otimes W_{G}\right)\right) \\
& =((\pi \times W) \otimes i)^{-}\left((\gamma \otimes i)^{-}\left(u^{*}\right)\left(1 \otimes W_{G}\right)\right) \\
& =\left[(\pi \otimes i)^{-}\left(u^{*}\right)\right]\left[((\pi \times W) \otimes i)^{-}\left(1 \otimes W_{G}\right)\right] \\
& =\left[(\pi \otimes i)^{-}\left(u^{*}\right)\right] W .
\end{aligned}
$$

Here we have used $(\pi \times W)^{-} \circ \gamma=\pi$ since $(\pi, W)$ is a $\gamma$-covariant pair. Hence $V=(\pi \otimes i)^{-}\left(u^{*}\right) W$ and we are done. 
4. Invariant ideals. It is a basic observation in the theory of crossed products by actions that if $I$ is an ideal in $A$ which is invariant under an automorphism group $\alpha: G \rightarrow$ Aut $A$, then the inclusion of $C_{c}(G, I)$ in $C_{c}(G, A)$ embeds $I \times{ }_{\alpha} G$ as an ideal in $A \times{ }_{\alpha} G$, and the quotient map $A \rightarrow A / I$ induces an isomorphism of $\left(A \times{ }_{\alpha} G\right) /\left(I \times{ }_{\alpha} G\right)$ onto $(A / I) \times_{\alpha} G$ (for example, see [8, Proposition 12]). In this section we describe corresponding results for coactions. We have run into a few technical problems here-it is not even clear which ideals we should describe as invariant (see Remarks 4.2) - but at least everything seems to work when $G$ is amenable. We also make use of the nondegeneracy condition for coactions-something which was not necessary in $\S \S 2$ and 3 . When we come to apply these general results in $\S \S 5$ and 6 , the special nature of the coactions involved there will allow us to circumvent the amenability hypothesis.

We recall that if $I$ is an ideal in $A$, then by Lemma 1.4, restriction is an isomorphism of $\left\{m \in \tilde{M}\left(A \otimes C_{r}^{*}(G)\right) \mid x m, m x \in I \otimes C_{r}^{*}(G)\right.$ for $\left.x \in A \otimes C_{r}^{*}(G)\right\}$ onto $\tilde{M}\left(I \otimes C_{r}^{*}(G)\right)$, so that we can view $\tilde{M}\left(I \otimes C_{r}^{*}(G)\right)$ as a subalgebra of $\tilde{M}\left(A \otimes C_{r}^{*}(G)\right)$.

4.1. Definition. Let $\delta$ be a coaction of $G$ on $A$, and let $I$ be an ideal in $A$. We shall say $I$ is invariant under $\delta$ if

(a) $\delta(I) \subseteq \tilde{M}\left(I \otimes C_{r}^{*}(G)\right)$ and

(b) $\left.\delta\right|_{I}$ is a coaction.

4.2. REMARKs. (1) If $\delta$ is nondegenerate and $I$ is invariant under $\delta$, then $\left.\delta\right|_{I}$ is also nondegenerate. For, if $x \in I \otimes C_{r}^{*}(G)$, then the nondegeneracy of $\delta$ allows us to approximate $x$ by an element of the form $y=\sum \delta\left(a_{i}\right)\left(1 \otimes \lambda\left(g_{i}\right)\right)$ by [12, Theorem 5]. Letting $\left\{f_{k}\right\}$ be an approximate identity for $I$, we see that by condition (b), $x$ is approximately $\delta\left(f_{k}\right) x$ which in turn is approximately $\delta\left(f_{k}\right) y$ which equals $\sum_{i} \delta\left(f_{k} a_{i}\right)\left(1 \otimes \lambda\left(g_{i}\right)\right)$. Thus, $\left.\delta\right|_{I}$ is nondegenerate by [12, Theorem 5].

(2) We do not know whether $\left.\delta\right|_{I}$ is always a coaction when (a) is satisfied; however, Proposition 4.3 shows that this is the case if $G$ is amenable. The proof of that proposition shows that, in general, all we need to check is the condition on approximate identities.

(3) Katayama [11, p. 252] has described an ideal $I$ as $\delta$-invariant if $S_{f}(\delta(a)) \in I$ for all $a \in I$ and $f \in B_{r}(G)$. It is not hard to see that the slice maps on $\tilde{M}(I \otimes B)$ are just restrictions of the slice maps on $\tilde{M}(A \otimes B)$, so that if $I$ is invariant as in Definition 4.1, it is also $\delta$-invariant in Katayama's sense.

Now suppose $I$ is $\delta$-invariant in Katayama's sense and let $b \in I$. Then $\delta(b) \in$ $\tilde{M}\left(I \otimes C_{r}^{*}(G)\right)$ if and only if $\delta(b) x, x \delta(b) \in I \otimes C_{r}^{*}(G)$ for all $x \in A \otimes C_{r}^{*}(G)$. If $a \in A$ and $\lambda(g) \in C_{r}^{*}(G)$, then

$$
S_{f}(\delta(b)(a \otimes \lambda(g)))=S_{f \cdot \lambda(g)}(\delta(b)) a \in I .
$$

Thus (a) will be satisfied if

$$
S_{f}(y) \in I \text { for all } f \in B_{r}(G) \text { implies } y \in I \otimes C_{r}^{*}(G) .
$$

But this is the slice map property for $\left(C_{r}^{*}(G), A, I\right)$ which is known to be false in general. It does hold if $C_{r}^{*}(G)$ is nuclear, and even more generally [2]. In particular, if $G$ is amenable, $(*)$ is valid so that $\delta(b) \in \tilde{M}\left(I \otimes C_{r}^{*}(G)\right)$ and so that $I$ is 
invariant in our sense by Proposition 4.3. There are nonamenable groups for which (*) holds (for example $G=\mathrm{SL}_{2}(\mathbf{C})$, for which $C_{r}^{*}(G)$ is type I and hence nuclear), and then the invariance of $I$ depends on condition (b) - see the preceding remark. Of course, if $G$ is abelian and $\delta$ corresponds to the action $\alpha$ of $\hat{G}$, then $I$ is invariant for $\delta$ if and only if it is invariant under $\alpha$ in the usual sense.

4.3. Proposition. Suppose $\delta$ is a (nondegenerate) coaction of a locally compact amenable group $G$ on a $C^{*}$-algebra $A$. If $I$ is an ideal in $A$ such that $\delta(I)$ is contained in $\tilde{M}\left(I \otimes C_{r}^{*}(G)\right)$, then $\left.\delta\right|_{I}$ is a (nondegenerate) coaction.

Our proof of this result depends (in part) on the following lemma, which is a consequence of the definitions of the various extensions in Lemma 1.1.

4.4. Lemma. Let $J, K$ be ideals in $C^{*}$-algebras $B, D$ respectively, let $r$ denote the restriction maps on multipliers, and suppose $\phi: B \rightarrow M(D)$ is a homomorphism such that there are approximate identities

(1) $\left\{e_{j}\right\}$ for $B$ with $\phi\left(e_{j}\right) \rightarrow 1$ strictly in $M(D)$;

(2) $\left\{f_{k}\right\}$ for $J$ with $r\left(\phi\left(f_{k}\right)\right) \rightarrow 1$ strictly in $M(K)$.

Then, the following diagram commutes:

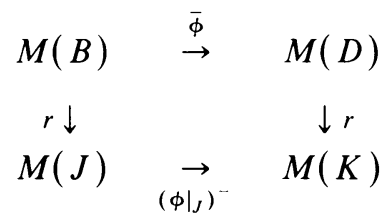

Proof of Proposition 4.3. We have to prove that if $\left\{f_{j}\right\}$ is an approximate identity for $I$, then $\delta\left(f_{j}\right) \rightarrow 1$ strictly in $M\left(I \otimes C_{r}^{*}(G)\right)$; this will be easy once we have shown that $I \otimes C_{r}^{*}(G)$ is the closed span of $\delta(I)\left(1 \otimes C_{r}^{*}(G)\right)$. First of all it is established in the proof of [13, Lemma 3.8] that $S_{1}(\delta(a))=a$ for all $a \in A$, where 1 is the constant function in $B(G)=B_{r}(G)$ since $G$ is amenable. Now choose $g \in C_{c}(G)$ with $\langle\lambda(g), 1\rangle=1=\int_{G} g(t) d t$. Then we have $1 \cdot \lambda(g)=1$ in $B_{r}(G)$. Thus, if $\left\{h_{i}\right\}$ is a net in $A(G) \cap C_{c}(G)$ converging weak-* to 1 , then we get for any $a \in A$ :

$$
S_{h_{i} \cdot \lambda(g)}(\delta(a))=S_{h_{i}}(\delta(a)(1 \otimes \lambda(g))) \rightarrow S_{1}(\delta(a)(1 \otimes \lambda(g)))=S_{1}(\delta(a))=a .
$$

In particular, it follows that we can approximate any $a \in I$ by elements of the form $S_{u} \circ \delta\left(S_{h} \circ \delta(a)\right)$, for $u, h \in A(G) \cap C_{c}(G)$. Now, for such $u, h$ and $a \in A, \lambda(g) \in$ $C_{r}^{*}(G)$, Katayama has shown that if $\tilde{u}(s)=u\left(s^{-1}\right)$, then

$$
\begin{aligned}
& S_{u} \circ \delta\left(S_{h} \circ \delta(a)\right) \otimes \lambda(g) \\
& \quad=\lim _{v} \int_{G} \tilde{u}(s) \delta\left(S_{v \cdot \lambda(s)} \circ \delta\left(S_{h} \circ \delta(a)\right)\right)(1 \otimes \lambda(s) \lambda(g)) d s,
\end{aligned}
$$

where $v$ also belongs to $A(G) \cap C_{c}(G)$, and the limit is taken as the measure $v(s) d s$ converges to the point mass at the identity of $G$ [12, Proof of Lemma 3]. If $a \in I$, then these integrands belong to $\delta(I)\left(1 \otimes C_{r}^{*}(G)\right)$ so we have established that the span of $\delta(I)\left(1 \otimes C_{r}^{*}(G)\right)$ is dense in $I \otimes C_{r}^{*}(G)$ as claimed. It is now clear that 
$\left\{\delta\left(f_{j}\right)\right\}$ converges strictly to 1 in $M\left(I \otimes C_{r}^{*}(G)\right)$. This enables us to make sense of the coaction identity for $\left.\delta\right|_{I}$ using Lemma 1.1, and a few applications of Lemma 4.4 show that it is satisfied. Thus, $\left.\delta\right|_{I}$ satisfies (a) and (b), and $I$ is invariant.

Suppose $I$ is an ideal in $A$ which is invariant under a coaction $\delta$ of $G$. Then we can form the crossed product $I \times{ }_{\delta} G$, and we would like to embed it as an ideal in $A \times{ }_{\delta} G$. The generators for $I \times_{\delta} G$ lie in the algebra $\tilde{M}\left(I \otimes K\left(L^{2}(G)\right)\right)$, which by Lemma 1.4 we can view as a subalgebra of $\tilde{M}(A \otimes K)$. When we do this, we can regard $I \times{ }_{\delta} G$ as the $C^{*}$-subalgebra of $A \times_{\delta} G$ generated by elements of the form $\delta(a)\left(1 \otimes M_{f}\right)$ for $a \in I, f \in C_{0}(G)$. We shall now show that this subalgebra is, in fact, an ideal.

4.5. Proposition. Let $\delta$ be a coaction of $G$ on $A$ and suppose $I \triangleleft A$ is invariant under $\delta$. Then $I \times{ }_{\delta} G$ is an ideal in $A \times{ }_{\delta} G$.

Proof. Since $\left.\delta\right|_{I}$ is a coaction (by definition) we have that by Lemma 2.5 any $x \in I \times{ }_{\delta} G$ can be factored: $x=\delta(a) y$ where $a \in I$ and $y \in I \times{ }_{\delta} G$. Now, if $\left(1 \otimes M_{f}\right) \delta(b)$ is a generator of $A \times{ }_{\delta} G$, then clearly

$$
\left(1 \otimes M_{f}\right) \delta(b) x=\left(I \otimes M_{f}\right) \delta(b a) y \in I \times{ }_{\delta} G .
$$

Another application of Lemma 2.5 shows that generators of the form $\delta(b)\left(1 \otimes M_{f}\right)$ are also left multipliers of $I \times{ }_{\delta} G$ so that $I \times{ }_{\delta} G$ is a left ideal in $A \times{ }_{\delta} G$, and hence an ideal since it is a ${ }^{*}$-subalgebra.

We would now like to construct a canonical coaction of $G$ on $A / I$, where $I$ is an invariant ideal, and prove that the crossed product $(A / I) \times_{\delta} G$ is isomorphic to $\left(A \times{ }_{\delta} G\right) /\left(I \times{ }_{\delta} G\right)$. Notice that we have again run into a technical problem in the nonamenable case.

4.6. LEMMA. Let $\delta$ be a coaction of $G$ on $A$, and suppose $I$ is an ideal invariant under $\delta$. Let $\theta: A \rightarrow A / I$ be the quotient map. Then, defining

$$
\delta^{I}(\theta(a))=(\theta \otimes i)^{-}(\delta(a)) \quad \text { for } a \in A
$$

yields a homomorphism $\delta^{I}$ of $A / I$ into $\tilde{M}\left((A / I) \otimes C_{r}^{*}(G)\right)$ which satisfies all the requirements for a coaction, except possibly injectivity. If $G$ is amenable, then $\delta^{I}$ is a (nondegenerate) coaction.

Proof. We first observe that, by an application of Lemma 1.4, the kernel of $(\theta \otimes i)^{-}$contains $\tilde{M}\left(I \otimes C_{r}^{*}(G)\right)$ so that $\delta^{I}$ is well defined. It is straightforward to verify that $\delta^{I}(\theta(a))$ belongs to $\tilde{M}\left((A / I) \otimes C_{r}^{*}(G)\right)$, that $\delta^{I}\left(\theta\left(e_{i}\right)\right)$ converges to 1 strictly for every approximate identity $\left\{e_{i}\right\}$ of $A$, and that $\delta^{I}$ satisfies the coaction condition (a cubical diagram chase). Now, if $G$ is amenable, then the kernel of $(\theta \otimes i)^{-}$restricted to $\tilde{M}\left(A \otimes C_{r}^{*}(G)\right)$ is just $\tilde{M}\left(I \otimes C_{r}^{*}(G)\right)$ since $C_{r}^{*}(G)$ is $C^{*}$ exact in the sense that $\operatorname{ker}(\theta \otimes i)=\operatorname{ker} \phi \otimes C_{r}^{*}(G)$ for all $\phi: B \rightarrow \phi(B)$. Now, if $\delta^{I}(\theta(a))=0$, then $\delta(a)$ is in $\tilde{M}\left(I \otimes C_{r}^{*}(G)\right)$ and so $a=S_{1}(\delta(a)) \in I$. Thus, $\theta(a)=0$ and $\delta^{I}$ is injective.

4.7. Remarks. (1) If $\delta$ is nondegenerate and $\delta^{I}$ is injective, then $\delta^{I}$ is also a nondegenerate coaction. This follows from [12, Theorem 5], since condition (ii) of that theorem passes to the quotient under $\theta \otimes i$. 
(2) The proof of the lemma shows that $\delta^{I}$ will be injective whenever the kernel of $(\theta \otimes i)^{-}$is $\tilde{M}\left(I \otimes C_{r}^{*}(G)\right)$ and $\delta(a) \in \tilde{M}\left(I \otimes C_{r}^{*}(G)\right)$ implies $a \in I$.

4.8. Proposition. Let $\delta$ be a nondegenerate coaction of $G$ on $A$ and suppose $I$ is an invariant ideal such that $\delta^{I}: A / I \rightarrow \tilde{M}\left((A / I) \otimes C_{r}^{*}(G)\right)$ is also a nondegenerate coaction. Then, the quotient map $\theta \otimes i$ of $A \otimes K\left(L^{2}(G)\right)$ onto $(A / I) \otimes K\left(L^{2}(G)\right)$ induces an isomorphism of $\left(A \times_{\delta} G\right) /\left(I \times{ }_{\delta} G\right)$ on $(A / I) \times_{\delta^{\prime}} G$. The transpose of this map gives the homeomorphism $\pi \times W \mapsto(\pi \circ \theta) \times W$ of $\left((A / I) \times{ }_{\delta^{\prime}} G\right)^{\wedge}$ onto the closed set $F=\left\{\pi \times W|\pi|_{I}=0\right\}$ in $\left(A \times_{\delta} G\right)^{\wedge}$.

Our proof of this will use a slightly more precise version of the duality theorem of Katayama [12], which we now discuss. As in $\$ 1$ we let $\rho$ denote the right regular representation of $G$ on $L^{2}(G)$ : i.e., $(\rho(s) \xi)(t)=\Delta^{1 / 2}(s) \xi(t s)$ for $s, t \in G$ and $\xi \in L^{2}(G)$. Now, each operator of the form $1 \otimes \rho(s)$ commutes with $A \otimes C_{r}^{*}(G)$, and hence with $\delta(A)$. Moreover, $\rho(s) M_{f} \rho(s)^{*}$ is multiplication by the function $t \mapsto f(t s)$, so that $\hat{\delta}_{s}=\operatorname{Ad}(1 \otimes \rho(s))$ gives an automorphism group

$$
\hat{\delta}: G \rightarrow \operatorname{Aut}\left(A \times{ }_{\delta} G\right),
$$

called the dual action. If we define $V_{G} \in U\left(L^{2}(G \times G)\right)$ by

$$
\left(V_{G} \xi\right)(s, t)=\Delta^{1 / 2}(t) \xi\left(s t^{-1}, t\right)
$$

and define a faithful representation $\tilde{\pi}$ of $A \times{ }_{\delta} G$ on $L^{2}\left(G, L^{2}(G, H)\right)$ by

$$
(\tilde{\pi}(b) \xi)(s)=\hat{\delta}_{s}^{-1}(b)(\xi(s)) \text { for } b \in A \times_{\delta} G
$$

(where $A$ is concretely represented on $H$ ), then a routine calculation on the generators of $A \times{ }_{\delta} G$ shows that

$$
\tilde{\pi}(b)=\left(1 \otimes V_{G}\right)(b \otimes 1)\left(1 \otimes V_{G}^{*}\right)
$$

on $H \otimes L^{2}(G) \otimes L^{2}(G)$. Since $\tilde{\pi} \times(1 \otimes 1 \otimes \lambda)$ is a faithful representation of the reduced crossed product $\left(A \times_{\delta} G\right) \times_{\hat{\delta}, r} G$ [16, Theorem 7.7.5], we can identify it with the $C^{*}$-algebra generated by

$$
\tilde{\pi}\left(A \times_{\delta} G\right)\left(1 \otimes 1 \otimes C_{r}^{*}(G)\right) \subseteq \mathscr{B}\left(H \otimes L^{2}(G) \otimes L^{2}(G)\right) .
$$

Katayama just calls this the "crossed product."

Let $S$ be the unitary operator on $L^{2}(G \times G, H)$ defined by $(S \xi)(s, t)=$ $\Delta^{-1 / 2}(t) \xi\left(s, t^{-1}\right)$. The version of Katayama's theorem which we need says that

$$
\Phi=\operatorname{Ad}\left[S\left(1 \otimes W_{G}^{*}\right)\right] \circ(\delta \otimes i)
$$

is an isomorphism of $A \otimes K\left(L^{2}(G)\right)$ onto $\left(A \times_{\delta} G\right) \times_{\hat{\delta}, r} G$.

In fact, because $\delta$ is nondegenerate, $A \otimes K$ is generated by products of the form

$$
\delta(a)\left(1 \otimes M_{f}\right)(1 \otimes \rho(g)) .
$$

Katayama proves that $\Phi$ is an isomorphism of $A \otimes K$ onto $\left(A \times_{\delta} G\right) \times_{\hat{\delta}_{, r}} G$ by showing that

$$
\left\{\begin{array}{l}
\Phi(\delta(a))=\operatorname{Ad}\left[S\left(1 \otimes W_{G}^{*}\right)\right](\delta \otimes i(\delta(a))) \\
=S(\delta(a) \otimes 1) S^{*}=\delta(a) \otimes 1=\tilde{\pi}(\delta(a)), \\
\Phi\left(1 \otimes M_{f}\right)=\operatorname{Ad}\left[S\left(1 \otimes W_{G}^{*}\right)\right]\left(1 \otimes 1 \otimes M_{f}\right)=\tilde{\pi}\left(1 \otimes M_{f}\right), \\
\Phi(1 \otimes \rho(g))=\operatorname{Ad}\left[S\left(1 \otimes W_{G}^{*}\right)\right](1 \otimes 1 \otimes \rho(g))=1 \otimes 1 \otimes \lambda(g) .
\end{array}\right.
$$

(Warning: Katayama's $W$ is our $W_{G}^{*}$ - sorry!) 
Proof of Proposition 4.8. Let $\theta \times{ }_{\delta} G$ be the surjection of $A \times{ }_{\delta} G$ onto $(A / I) \times{ }_{\delta^{\prime}} G$ induced by $(\theta \otimes i)^{-}$on $M(A \otimes K)$; we shall use duality theory to identify its kernel. As $\theta \times{ }_{\delta} G$ intertwines the group actions $\hat{\delta}$ and $\left(\delta^{I}\right)^{\wedge}$, it induces a homomorphism $\left(\theta \times{ }_{\delta} G\right) \times_{\hat{\delta}, r} G$ of $\left(A \times_{\delta} G\right) \times_{\hat{\delta}, r} \cdot G: \rightarrow\left((A / I) \times \times_{\delta^{\prime}} G\right) \times{ }_{\left(\delta^{\prime}\right)^{\wedge}, r}$ $G$. Let $\left(\pi^{I}\right)^{\sim}$ be a realization of $(A / I) \times_{\delta^{\prime}} G$ on $H_{1} \otimes L^{2}(G) \otimes L^{2}(G)$ (where $A / I$ is concretely represented on $H_{1}$ ) and let $\Phi^{I}$ be Katayama's duality isomorphism for $\delta^{I}$. Then on generators, $\left(\theta \times{ }_{\delta} G\right) \times_{\hat{\delta}, r} G=(\theta \times G) \times G$ is given by

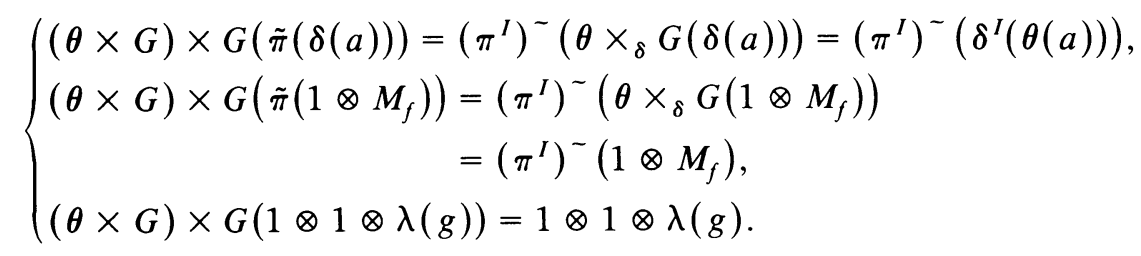

A straightforward computation using $(\dagger)$ and $(\dagger \dagger)$ shows that we have a commutative diagram:

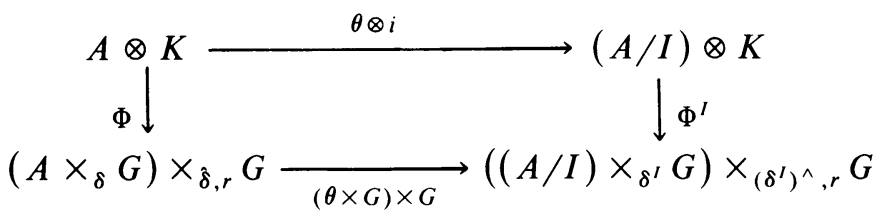

Thus, $\Phi$ maps $\operatorname{ker}(\theta \otimes i)=I \otimes K$ onto $\operatorname{ker}[(\theta \times G) \times G]$. By observing where $\phi$ carries generators, we see that $\operatorname{ker}[(\theta \times G) \times G]=\left(I \times{ }_{\delta} G\right) \times_{\hat{\delta}, r} G$. Now, if we had $I \times{ }_{\delta} G$ properly contained in $J=\operatorname{ker}\left(\theta \times{ }_{\delta} G\right)$, then since $J$ is invariant for $\hat{\delta}$, we would have $\left(I \times_{\delta} G\right) \times_{\hat{\delta}, r} G \subsetneq J \times_{\hat{\delta}, r} G$. But $J \times_{\hat{\delta}, r} G$ is contained in

$$
\operatorname{ker}[(\theta \times G) \times G]=\left(I \times_{\delta} G\right) \times_{\hat{\delta}, r} G
$$

so this is impossible, and we must have $\operatorname{ker}\left(\theta \times{ }_{\delta} g\right)=I \times{ }_{\delta} G$.

To see the last statement of the proposition, note first that the representations in $F$ are precisely those which vanish on $I \times{ }_{\delta} G$, so that composition with the quotient map is a homeomorphism of $F$ onto $\left(\left(A \times_{\delta} G\right) /\left(I \times{ }_{\delta} G\right)\right)^{\wedge}$. It is straightforward to check that $(\pi \times W) \circ\left(\theta \times{ }_{\delta} G\right)=(\pi \circ \theta) \times W$, and the result follows.

Our main source of invariant ideals will be the kernels of covariant pairs. Suppose $(\pi, W)$ is a covariant pair for $(A, G, \delta)$, and let $I=\operatorname{ker} \pi$. The covariance condition $(\pi \otimes i)^{-}(\delta(a))=W(\pi(a) \otimes 1) W^{*}$ shows that

$$
a \in I \Leftrightarrow \delta(a) \in \operatorname{ker}(\pi \otimes i)^{-} \Leftrightarrow \delta(a) x, x \delta(a) \in \operatorname{ker}(\pi \otimes i)
$$

for all $x \in A \otimes C_{r}^{*}(G)$. Hence $\delta$ will map $I$ into $\tilde{M}\left(I \otimes C_{r}^{*}(G)\right)$ if $\operatorname{ker}(\pi \otimes i)=$ $I \otimes C_{r}^{*}(G)$. This will happen if either $A$ or $C_{r}^{*}(G)$ is nuclear, in particular if $G$ is amenable. There is no reason to suspect that $C_{r}^{*}(G)$ is always $C^{*}$-exact [26].

We have proved

4.9. Proposition. Let $\delta$ be a (nondegenerate) coaction of an amenable locally compact group $G$ on a $C^{*}$-algebra $A$, and suppose that $(\pi, W)$ is a covariant pair of representations of $(A, G, \delta)$. Then $\operatorname{ker} \pi$ is invariant under $\delta, \delta$ induces a coaction of $G$ on $A / \operatorname{ker} \pi$, and $(\pi, W)$ defines a covariant pair of representations of $\left(A / \operatorname{ker} \pi, G, \delta^{\mathrm{ker} \pi}\right)$. 
4.10. Remark. If $G$ is nonamenable but $A$ is nuclear, then as above, $\delta: I \rightarrow$ $\tilde{M}\left(I \otimes C_{r}^{*}(G)\right)$. If $\left.\delta\right|_{I}$ satisfies the requirements on approximate identities, then $\left.\delta\right|_{I}$ is a nondegenerate coaction by Remarks 4.2(1), (2); furthermore, $\delta^{I}$ is then injective by Remark 4.7(2), and hence a nondegenerate coaction of $G$ on $A / I$ by Remark 4.7(1).

5. Pointwise and locally unitary coactions. We shall now show how our results on crossed products by coactions can be used to extend the results of $[18, \S 2]$ to nonabelian groups. We introduce first the notion of a pointwise unitary coaction, and show how, for such a coaction $\delta$ of $G$ on $A$, the spectrum of $A \times{ }_{\delta} G$ is naturally fibered over $\hat{A}$. In the case where the coaction is locally unitary, this fibration is a locally trivial principal $G$-bundle with respect to the dual action of $G$. We also show how to construct examples of the two types of coactions using duality theory.

The main results of [18] concern $C^{*}$-algebras with Hausdorff spectrum, and we shall assume from now on that our $C^{*}$-algebras have this property.

5.1. Definition. Let $\delta$ be a nondegenerate coaction of a locally compact group $G$ on a $C^{*}$-algebra $A$. If $\pi$ is a representation of $A$, and $W$ in $M\left(\pi(A) \otimes C_{r}^{*}(G)\right)$ is a corepresentation of $G$ such that $(\pi, W)$ is a covariant pair for $(A, G, \delta)$, then we shall say $\mathrm{W}$ implements $\delta$ in the representation $\pi$. If for each $\pi \in \hat{A}$ there is such a $W$, we call $\delta$ pointwise unitary.

5.2. Remark. We should check that when $G$ is abelian and $\hat{A}$ is Hausdorff, this definition agrees with that of a pointwise unitary action of $\hat{G}$ as in [18, p. 217]. Suppose the coaction $\delta$ corresponds to the action $\alpha: \hat{G} \rightarrow$ Aut $A$, and let $V_{G} \in$ $U\left(L^{2}(\hat{G} \times \hat{G})\right)$ be given by $V_{G} \xi(\gamma, \chi)=\xi(\gamma \chi, \chi)$. Then $W$ is a corepresentation of $G$ on $H_{\pi}=H$ if and only if the unitary $V=\operatorname{Ad}(1 \otimes \mathscr{F})(W)$ in $M\left(\pi(A) \otimes C_{0}(\hat{G})\right)$ satisfies

$$
(V \otimes 1)(V \otimes 1)^{\sigma}=\left(1 \otimes V_{G}\right)(V \otimes 1)\left(1 \otimes V_{G}^{*}\right)
$$

By [1], we can realize $V$ as a strictly continuous function from $\hat{G}$ to $U M(\pi(A))$ and then this condition says precisely that $\gamma \rightarrow V_{\gamma}$ is a strictly continuous unitary representation of $\hat{G}$ in $M(\pi(A))$. It is routine to check that $W$ implements $\delta$ in the representation $\pi$ if and only if $V$ implements $\alpha$ in the representation $\pi$. When $\hat{A}$ is Hausdorff, $\pi(A)=K(H)$ and so $\gamma \rightarrow V_{\gamma}$ is a strongly continuous unitary representation of $\hat{G}$ in $\mathscr{B}(H)$; so, the two definitions coincide in this case.

5.3. Propositron. Let $\delta$ be a pointwise unitary coaction of $G$ on a $C^{*}$-algebra $A$ with Hausdorff spectrum.

(1) For $f \in C_{b}(\hat{A})$ we have $\bar{\delta}(f)=f \otimes 1 \in M\left(A \times_{\delta} G\right)$ so that $\bar{\delta}$ embeds $C_{b}(\hat{A})$ $=Z M(A)$ into $Z M\left(A \times{ }_{\delta} G\right)$.

(2) If $N$ is a closed subset of $\hat{A}$, and $I=\bigcap\{\operatorname{ker} \pi \mid \pi \in N\}$, then $I$ is invariant under $\delta$, and $\delta^{I}$ is a nondegenerate coaction of $G$ on $A / I$.

(3) If $\pi \in \hat{A}$, then $\operatorname{ker} \pi$ is invariant under $\delta$ and $\delta^{\pi}=\delta^{\mathrm{ker} \pi}$ is a unitary coaction of $G$ on $\pi(A)$. 
Proof. Suppose $W_{\pi}$ implements $\delta$ in the representation $\pi \in \hat{A}$. Then, for $f \in C_{b}(\hat{A})$ we have

$$
\begin{aligned}
{\left[(\pi \otimes i)^{-} \circ \bar{\delta}(f)\right]\left[(\pi \otimes i)^{-} \circ \delta(a)\right] } & =(\pi \otimes i)^{-} \circ \delta(f a) \\
& =W_{\pi}(\pi(f a) \otimes 1) W_{\pi}^{*} \\
& =f(\pi) W_{\pi}(\pi(a) \otimes 1) W_{\pi}^{*} \\
& =f(\pi)(\pi \otimes i)^{-} \circ \delta(a) .
\end{aligned}
$$

Hence $(\pi \otimes i)^{-} \circ \bar{\delta}(f)=f(\pi) \cdot 1_{H_{\pi}} \otimes 1$ for each $\pi \in \hat{A}$. Letting $\rho=\bigoplus_{\pi \in \hat{A}} \pi$, which is a faithful representation of $A$, we get $(\rho \otimes i)^{-} \circ \bar{\delta}(f)=f \otimes 1$, where on the right-hand side we have identified $f$ with $\bar{\rho}(f)$. This implies (1) by the independence of $A \times{ }_{\delta} G$ on the (faithful) representation of $A$.

To establish (2), note that $I=\operatorname{ker} \bigoplus_{\pi \in N} \pi$ and that $\left(\oplus_{\pi \in N} \pi, \bigoplus_{\pi \in N} W_{\pi}\right)$ is a covariant pair. Hence, by Remark 4.10 , it will be enough to find an approximate identity $\left\{g_{k}\right\}$ for $I$ such that $\delta\left(g_{k}\right) \rightarrow 1$ strictly on $I \otimes C_{r}^{*}(G)$. Now, let $\left\{e_{i}\right\},\left\{f_{j}\right\}$ be approximate identifies for $A, C_{0}(\hat{A} \backslash N)$ respectively. Then $f_{j} \otimes 1$ and $r\left(\delta\left(e_{i}\right)\right)$ both converge to 1 strictly in $M\left(I \otimes C_{r}^{*}(G)\right)$ and therefore so does

$$
\left(f_{j} \otimes 1\right) r\left(\delta\left(e_{i}\right)\right)=r\left(\delta\left(f_{j} e_{i}\right)\right)=\delta\left(f_{j} e_{i}\right) \text {. }
$$

Since $f_{j} e_{i} \in I$ we can take $g_{j i}=f_{j} e_{i}$.

Part (3) follows easily from part (2).

We now want to see that the spectrum of the crossed product $A \times{ }_{\delta} G$ by a pointwise unitary coaction is fibered over $\hat{A}$ by the dual action $\hat{\delta}$ of $G$. Our proof is rather similar to that of the abelian version [18, Proposition 2.1]. In the next lemma $\delta$ is not assumed to be nondegenerate and $\hat{A}$ is not assumed to be Hausdorff.

5.4. LEMMA. If $(\pi, W)$ is a covariant pair for $(A, G, \delta)$, then so is $(\pi, W(1 \otimes \lambda(s)))$ for $s \in G$ and $(\pi \times W) \circ \hat{\delta}_{s}=\pi \times W(1 \otimes \lambda(s))$.

Proof. As $(\pi \times W) \circ \hat{\delta}_{s}$ is a representation of $A \times{ }_{\delta} G$ and as $\hat{\delta}_{s}$ (extended to $\left.M\left(A \times{ }_{\delta} G\right)\right)$ leaves $A$ pointwise fixed we see that $(\pi \times W) \circ \hat{\delta}_{s}=\pi \times V$ for some corepresentation $V$ of $G$, where $(\pi, V)$ is covariant. Now, for $f \in A(G)$

$$
\begin{aligned}
S_{f}(V) & =(\pi \times V)\left(1 \otimes M_{f}\right)=(\pi \times W) \circ \hat{\delta}_{s}\left(1 \otimes M_{f}\right) \\
& =(\pi \times W)\left(1 \otimes M_{f \cdot s}\right)=S_{f \cdot s}(W)=S_{f}(W(1 \otimes \lambda(s))) .
\end{aligned}
$$

One easily checks that $W(1 \otimes \lambda(s))$ is a corepresentation of $G$ and so by [14], $V=W(1 \otimes \lambda(s))$.

5.5. THEOREM. Let $\delta$ be a pointwise unitary coaction of a locally compact group $G$ on a $C^{*}$-algebra $A$ with Hausdorff spectrum.

(1) If $\pi \times W$ is an irreducible representation of $A \times{ }_{\delta} G$, then $\pi$ is an irreducible representation of $A$.

(2) If $\pi \in \hat{A}$ and $\left(\pi, W_{i}\right)$ are covariant for $i=1,2$, then there exists an $s \in G$ such that $W_{1}=W_{2}(1 \otimes \lambda(s))$. Furthermore, $(\pi \times W) \circ \hat{\delta}_{s}$ is not equivalent to $\pi \times W$ unless $s=e$.

(3) $A \times{ }_{\delta} G$ is a CCR (or liminal) $C^{*}$-algebra and the map $p: \pi \times W \rightarrow \pi$ is a continuous surjection of $\left(A \times_{\delta} G\right)^{\wedge}$ onto $\hat{A}$. We call $p$ the restriction map (of course, $\left(A \times{ }_{\delta} G\right)^{\wedge}$ need not be Hausdorff $)$. 
Proof. Let $\nu=\pi \times W \in\left(A \times{ }_{\delta} G\right)^{\wedge}$. Then by Proposition 5.3 we have for $f \in C_{0}(\hat{A})$

$$
\bar{\pi}(f)=\bar{\nu}(\bar{\delta}(f)) \in \bar{\nu}\left(Z M\left(A \times_{\delta} G\right)\right) \subseteq \nu\left(A \times_{\delta} G\right)^{\prime}=\mathbf{C} \cdot 1 .
$$

So, there exists $\pi_{1} \in \hat{A}$ such that $\bar{\pi}(f)=f\left(\pi_{1}\right)$ for all $f \in C_{0}(\hat{A})$. Since $\hat{A}$ is Hausdorff, ideals in $A$ are bijectively determined by ideals in $C_{0}(\hat{A})$ and are of the form $A I$ where $I$ is an ideal in $C_{0}(\hat{A})$. Thus, $\operatorname{ker} \pi=\operatorname{ker} \pi_{1}$ and so $\pi$ is a multiple of $\pi_{1}$ : we therefore assume that $\pi=1 \otimes \pi_{1}$ on $H \otimes K$.

Let $W_{1}$ be a corepresentation of $G$ on $K$ which implements $\delta$ in $\pi_{1}$. Then, for $a \in A$ we have

$$
\begin{aligned}
W\left(1 \otimes \pi_{1}(a) \otimes 1\right) W^{*}=W(\pi(a) \otimes 1) W^{*}=(\pi \otimes i)^{-}(\delta(a)) \\
=\left(\left(1 \otimes \pi_{1}\right) \otimes i\right)^{-}(\delta(a))=\left(1 \otimes W_{1}\right)\left(1 \otimes \pi_{1}(a) \otimes 1\right)\left(1 \otimes W_{1}^{*}\right) .
\end{aligned}
$$

Since $\pi_{1}$ is irreducible, we see that

$$
\left(1 \otimes W_{1}^{*}\right) W=\sum x_{i} \otimes 1_{K} \otimes y_{i} \quad \text { (approximately, weak operator), }
$$

where $U=\sum x_{i} \otimes y_{i}$ is unitary in $\mathscr{B}(H) \bar{\otimes} L(G)$. To see that $U$ defines a corepresentation of $G$ on $H$, we first show that $(W \otimes 1)$ commutes with $\left(1 \otimes W_{1}^{*} \otimes 1\right)^{\sigma}$ :

$$
\begin{aligned}
\left(1 \otimes W_{1}^{*} \otimes 1\right)^{\sigma}(W \otimes 1) & =\left(1 \otimes W_{1}^{*} \otimes 1\right)^{\sigma}\left(1 \otimes W_{1} \otimes 1\right)\left(\sum x_{i} \otimes 1_{K} \otimes y_{i} \otimes 1\right) \\
& =\left(1 \otimes W_{1} \otimes 1\right)\left(1 \otimes W_{1}^{*} \otimes 1\right)^{\sigma}\left(\sum x_{i} \otimes 1_{K} \otimes y_{i} \otimes 1\right) \\
& =\left(1 \otimes W_{1} \otimes 1\right)\left(\sum x_{i} \otimes 1_{K} \otimes y_{i} \otimes 1\right)\left(1 \otimes W_{1}^{*} \otimes 1\right)^{\sigma} \\
& =(W \otimes 1)\left(1 \otimes W_{1}^{*} \otimes 1\right)^{\sigma},
\end{aligned}
$$

where the first commutation is due to the fact that $\left(1 \otimes W_{1}^{*}\right)$ is a corepresentation of $G$ and so $\left(1 \otimes W_{1}^{*} \otimes 1\right)^{\sigma}$ commutes with $\left(1 \otimes W_{1}^{*} \otimes 1\right)$ and therefore with $\left(1 \otimes W_{1} \otimes 1\right)$. The second commutation is clear. Now, let $\tau$ denote the flip isomorphism of $\mathscr{B}(K) \bar{\otimes} \mathscr{B}(H)$ with $\mathscr{B}(H) \bar{\otimes} \mathscr{B}(K)$ and compute:

$$
\begin{aligned}
\left\{1_{K}\right. & \left.\otimes\left[(U \otimes 1)(U \otimes 1)^{\sigma}\right]\right\}^{\tau}=\left[\left(1_{K} \otimes U \otimes 1\right)\left(1_{K} \otimes U \otimes 1\right)^{\sigma}\right]^{\tau} \\
& =\left(1_{K} \otimes U \otimes 1\right)^{\tau}\left[\left(1_{K} \otimes U \otimes 1\right)^{\tau}\right]^{\sigma} \\
& =\left(\sum_{i} 1_{K} \otimes x_{i} \otimes y_{i} \otimes 1\right)^{\tau}\left[\left(\sum_{j} 1_{K} \otimes x_{j} \otimes y_{j} \otimes 1\right)^{\tau}\right]^{\sigma} \\
& =\left(\sum_{i} x_{i} \otimes 1_{K} \otimes y_{i} \otimes 1\right)\left(\sum_{j} x_{j} \otimes 1_{K} \otimes y_{j} \otimes 1\right)^{\sigma} \\
& =\left(1 \otimes W_{1}^{*} \otimes 1\right)(W \otimes 1)\left(1 \otimes W_{1}^{*} \otimes 1\right)^{\sigma}(W \otimes 1)^{\sigma} \\
& =\left(1 \otimes W_{1}^{*} \otimes 1\right)\left(1 \otimes W_{1}^{*} \otimes 1\right)^{\sigma}(W \otimes 1)(W \otimes 1)^{\sigma} \\
& =i \otimes \delta_{G}\left(1 \otimes W_{1}^{*}\right) i \otimes \delta_{G}(W)=i \otimes \delta_{G}\left(\left(1 \otimes W_{1}^{*}\right) W\right)=i \otimes \delta_{G}\left(\left(1_{K} \otimes U\right)^{\tau}\right) \\
& =\left\{1_{K} \otimes \delta_{G}(U)\right\}^{\tau} .
\end{aligned}
$$

Thus, $U$ is a corepresentation of $G$ on $H$. 
Now,

$$
\nu\left(\delta(a)\left(1 \otimes M_{f}\right)\right)=\pi(a) S_{f}(W)=\left(1_{H} \otimes \pi_{1}(a)\right) S_{f}\left(\left(1_{H} \otimes W_{1}\right)\left(1_{K} \otimes U\right)^{\tau}\right)
$$

and (except for an interchange in order) we see that $f \mapsto S_{f}(W)$ is just Nakagami and Takesaki's *-product of the representations $f \mapsto S_{f}\left(W_{1}\right)$ and $f \mapsto S_{f}(U)$ [14]. By formula A.13 of [14, Theorem A.3], we see that $S_{f}(U)$ commutes with $S_{g}(W)$ for all $f$ and $g$. As $S_{f}(U)$ clearly commutes with $\pi(a)=1_{H} \otimes \pi_{1}(a)$, we see that $H$ must be one-dimensional since $\nu=\pi \times W$ is irreducible. Thus, $\pi=\pi_{1}$ and we have part (1).

Now, if $\left(\pi, W_{1}\right)$ and $\left(\pi, W_{2}\right)$ are both covariant pairs for the irreducible representation $\pi$, then as above, $W_{2}^{*} W_{1}=1 \otimes U$ where $U$ is a unitary in $L(G)$. By the previous computations $U$ defines a corepresentation of $G$ on a one-dimensional space. Since $A(G)^{\wedge}=G$, there is an $s \in G$ so that $S_{f}(U)=f(s)$ for all $f \in A(G)$. As $\lambda(s)$ is a corepresentation of $G$ yielding the representation $f \mapsto f(s)$, we must have $U=\lambda(s)$ and the first part of (2) follows. It is easy to see that $\pi \times W_{1} \simeq$ $\pi \times W_{2}$ implies $W_{1}=W_{2}$ and so (2) follows from Lemma 5.4.

Now, let $\pi \times W \in\left(A \times{ }_{\delta} G\right)^{\wedge}$, and let $\mu$ be the representation of $A(G)$ corresponding to $W$. Then $(\pi \times W)\left(\delta(a)\left(1 \otimes M_{f}\right)\right)=\pi(a) \mu(f)$ is compact since $\pi(a)$ is compact. Hence $A$ is CCR. Part (1) shows that $p$ takes values in $\hat{A}$; it is surjective since $\delta$ is pointwise unitary; and it is continuous by a straightforward argument (see, for example, the last part of [18, Proposition 2.1]).

5.6. Definition. Let $\delta$ be a nondegenerate coaction of $G$ on $A$ where $\hat{A}$ is Hausdorff. We shall say $\delta$ is locally unitary if each $\pi \in \hat{A}$ has a neighborhood $N$ such that $I=\bigcap_{\pi \in N} \operatorname{ker} \pi$ is invariant and $\delta^{I}$ is a unitary coaction of $G$ on $A / I$.

5.7. REMARKS. (1) A locally unitary coaction is automatically pointwise unitary. To see this let $\pi, N, I$ be as in the definition and suppose $W$ implements $\delta^{I}$. Then $\pi$ defines a representation $\pi^{I}$ of $A / I$, and $\left(\pi,\left(\pi^{I} \otimes i\right)^{-}(W)\right)$ is a covariant pair for $(A, G, \delta)$.

(2) Suppose $G$ is abelian and $\delta$ corresponds to the action $\alpha$ of $\hat{G}$ on $A$. The argument of Remark 5.2 shows that $\delta$ is unitary if and only if $\alpha$ is implemented by a strictly continuous homomorphism $u$ of $G$ into $U M(A)$. To see that $\delta$ is locally unitary if and only if $\alpha$ is locally unitary as in [18, p. 217], we realize $A$ as the section algebra $\Gamma_{0}(E)$ of a continuous field of elementary $C^{*}$-algebras over $\hat{A}$. By shrinking neighborhoods we can see that $\alpha$ is locally unitary as in [18] if and only if each $\pi \in \hat{A}$ has a compact neighborhood $N$ such that the induced action of $\hat{G}$ on $\Gamma\left(\left.E\right|_{N}\right)$ is implemented by $u: \hat{G} \rightarrow U M\left(\Gamma\left(\left.E\right|_{N}\right)\right)$. Since the quotient of $A$ by $\bigcap_{\pi \in N} \operatorname{ker} \pi$ is canonically isomorphic to $\Gamma\left(\left.E\right|_{N}\right)$, this is equivalent, by our earlier observation, to saying $\delta$ is locally unitary.

(3) If $G$ is a compact Lie group, then a pointwise unitary coaction of $G$ is locally unitary if and only if $\left(A \times_{\delta} G\right)^{\wedge}$ is Hausdorff. One direction follows easily from Theorem 5.9. On the other hand, if $\left(A \times_{\delta} G\right)^{\wedge}$ is Hausdorff, then by a result of Gleason [6], the map $\left(A \times{ }_{\delta} G\right)^{\wedge} \rightarrow \hat{A}$ is a locally trivial $G$-bundle which implies by Proposition 5.14 that the dual coaction of $G$ on $\left(A \times_{\delta} G\right) \times{ }_{\alpha} G$ is locally unitary. But $\left(A \times_{\delta} G\right) \times_{\alpha} G \cong A \otimes K\left(L^{2}(G)\right)$ by Katayama's duality theorem [12] and the dual coaction on $A \otimes K\left(L^{2}(G)\right)$ restricted to $A \otimes 1$ is just $\delta \otimes i$ which implies that $\delta$ is locally unitary. 
We could have defined locally unitary coactions in a manner which was more obviously an extension of the abelian case, but our present definition seems slightly more natural and convenient. Of course, if we were working with a more general class of $C^{*}$-algebras we would have to be more careful, even in the abelian case.

5.8. Definition. We recall the definition of pull-back $C^{*}$-algebras from [21]. Let $p: X \rightarrow \hat{A}$ be continuous so that both $C_{0}(X)$ and $A$ are $C_{b}(\hat{A})$-modules in a natural way. Then $p^{*} A$, the pull-back of $A$ along $p$, is the "balanced" tensor product $C_{0}(X) \otimes_{C_{b}(\hat{A})} A$, which is just the quotient of $C_{0}(X) \otimes A$ by the ideal generated by $\left\{f b \otimes a-b \otimes f a \mid f \in C_{b}(\hat{A}), b \in C_{0}(X), a \in A\right\}$. We refer the reader to [21] for motivation and further properties of this notion.

5.9. THEOREM. Let $\delta$ be a locally unitary coaction of a locally compact group $G$ on a $C^{*}$-algebra $A$ with Hausdorff spectrum. Then the restriction map $p:\left(A \times{ }_{\delta} G\right)^{\wedge} \rightarrow \hat{A}$ and the dual action of $G$ on $A \times{ }_{\delta} G$ make $\left(A \times{ }_{\delta} G\right)^{\wedge}$ into a locally trivial principal G-bundle over $\hat{A}$. In fact, if $N$ is a compact subset of $\hat{A}, I=\bigcap\{\operatorname{ker} \pi \mid \pi \in N\}$, and $\delta^{I}$ is implemented by $W \in M\left((A / I) \otimes C_{r}^{*}(G)\right)$, then

$$
\Psi:(\pi, s) \mapsto\left[\pi \times\left(\pi^{I} \otimes i\right)^{-}(W)\right] \circ \hat{\delta}_{s}
$$

is a G-homeomorphism of $N \times G$ onto $p^{-1}(N)$. Furthermore, the map

$$
\Phi: C_{0}\left(\left(A \times{ }_{\delta} G\right)^{\wedge}\right) \otimes A \rightarrow M\left(A \times_{\delta} G\right)
$$

given by $\Phi(f \otimes a)=f \delta(a)$ induces an isomorphism of $p^{*} A$ onto $A \times{ }_{\delta} G$.

Proof. Let $\gamma$ be the trivial coaction of $G$ on $A / I$, so that $\gamma=\operatorname{Ad} W^{*} \circ \delta$. Now, $(A / I) \times{ }_{\gamma} G=A / I \otimes C_{0}(G)$ and so $\left((A / I) \times{ }_{\gamma} G\right)^{\wedge}=N \times G$, where the pair $(\pi, s)$ corresponds to the representation $\pi^{I} \times(1 \otimes \lambda(s))$ of $(A / I) \times{ }_{\gamma} G$. By Proposition 3.9 the isomorphism $\operatorname{Ad} W^{*}:(A / I) \times{ }_{\delta^{\prime}} G \rightarrow(A / I) \times{ }_{\gamma} G$ carries $\pi^{I} \times(1 \otimes \lambda(s))$ to $\pi^{I} \times\left[\left(\pi^{I} \otimes i\right)^{-}(W)(1 \otimes \lambda(s))\right]$. Now,

$$
\begin{aligned}
\pi^{I} \times & {\left[\left(\pi^{I} \otimes i\right)^{-}(W)(1 \otimes \lambda(s))\right]\left(\delta^{I}(a)\left(1 \otimes M_{f}\right)\right) } \\
& =\pi^{I}(a) S_{f}\left[\left(\pi^{I} \otimes i\right)^{-}(W)(1 \otimes \lambda(s))\right] \\
& =\pi^{I}(a) S_{f \cdot s}\left(\left(\pi^{I} \otimes i\right)^{-}(W)\right) \\
& =\left(\pi^{I} \times\left(\pi^{I} \otimes i\right)^{-}(W)\right)\left(\delta^{I}(a)\left(1 \otimes M_{f \cdot s}\right)\right) \\
& =\left[\left(\pi^{I} \times\left(\pi^{I} \otimes i\right)^{-}(W)\right) \circ \hat{\delta}_{s}\right]\left(\delta^{I}(a)\left(1 \otimes M_{f}\right)\right) .
\end{aligned}
$$

Thus, $(\pi, s) \rightarrow\left(\pi^{I} \times\left(\pi^{I} \otimes i\right)^{-}(W)\right) \circ \hat{\delta}_{s}$ is a $G$-homeomorphism of $N \times G$ onto $\left((A / I) \times{ }_{\delta^{\prime}} G\right)^{\wedge}$. An application of Proposition 4.8 completes the proof of the first part of the theorem.

For the second part of the theorem, we first have to establish that the range of $\Phi$ is contained in $A \times{ }_{\delta} G$. By a partition of unity argument, it will be enough to show that $\Phi(f \otimes a) \in A \times{ }_{\delta} G$ when the support of $f$ is contained in the interior of $p^{-1}(N)$ with $N$ as above. But $\Psi: N \times G \rightarrow p^{-1}(N) \subseteq\left(A \times{ }_{\delta} G\right)^{\wedge}$ and any function $f \circ \Psi$ on $N \times G$ is approximately a finite sum of tensors $g \otimes h$, where $g \in C_{0}(N), h \in C_{0}(G)$, so we can assume $f=(g \otimes h) \circ \Psi^{-1}$. In this case

$$
\Phi(f \otimes a)=f \delta(a)=\delta(g a)\left(1 \otimes M_{h}\right) \in A \times{ }_{\delta} G
$$

as required. 
Now, for $\pi \times W \in\left(A \times{ }_{\delta} G\right)^{\wedge}$ we have $(\pi \times W)(\Phi(f \otimes a))=(\pi \times W)(f \delta(a))$ $=f(\pi \times W) \pi(a)$ so that the transpose map $\hat{\Phi}$ on spectra is just $\hat{\Phi}(\pi \times W)=$ $(\pi \times W, \pi) \in\left[C_{0}\left(\left(A \times{ }_{\delta} G\right)^{\wedge}\right) \otimes A\right]^{\wedge}$. Hence, the kernel of $\Phi$ is the ideal in $C_{0}\left(\left(A \times{ }_{\delta} G\right)^{\wedge}\right) \otimes A$ determined by the closed set $\{(\pi \times W, \pi) \mid \pi \times W \in$ $\left.\left(A \times{ }_{\delta} G\right)^{\wedge}\right\}$. That is, $\operatorname{ker} \Phi=\bigcap\left\{\operatorname{ker}\left(\varepsilon_{\pi \times W} \otimes \pi\right) \mid \pi \times W \in\left(A \times{ }_{\delta} G\right)^{\wedge}\right\}$. This is easily seen to be the "balancing" ideal for the actions of $C_{0}(\hat{A})$ on $C_{0}\left(\left(A \times{ }_{\delta} G\right)^{\wedge}\right)$ and $A$, and so $\Phi$ defines an injection of $p^{*} A$ into $A \times{ }_{\delta} G$. It is easy to see that $\Phi\left(p^{*} A\right)$ vanishes at no point in $\left(A \times_{\delta} G\right)^{\wedge}$ and, in fact, is irreducible at each such point: thus by $[3,11.1 .4] \Phi\left(p^{*} A\right)=A \times{ }_{\delta} G$.

5.10. Corollary. Let $\delta$ be a locally unitary coaction of $G$ on a continuous trace $C^{*}$-algebra $A$, and let $p:\left(A \times{ }_{\delta} G\right)^{\wedge} \rightarrow \hat{A}$ be the restriction map. Then $A \times{ }_{\delta} G$ also has continuous trace, and its Dixmier-Douady class is given by $\delta\left(A \times{ }_{\delta} G\right)=p^{*}(\delta(A))$. ( Here $\delta(A)$ denotes the Dixmier-Douady class.)

Proof. This follows immediately from the theorem and [21, Proposition 1.4].

5.11. TheOREM. Let $\delta$ and $\gamma$ be locally unitary coactions of $G$ on the $C^{*}$-algebra A, and suppose $\hat{A}$ is Hausdorff. Then $\delta$ and $\gamma$ are exterior equivalent if and only if the G-bundles, $\left(A \times{ }_{\delta} G\right)^{\wedge} \rightarrow \hat{A}$ and $\left(A \times_{\gamma} G\right)^{\wedge} \rightarrow \hat{A}$, are isomorphic as G-bundles over $\hat{A}$.

Proof. Let $u$ be a $\delta$-cocycle so that by Theorem 2.9 Ad $u: A \times{ }_{\delta} G \rightarrow A \times{ }_{\gamma} G$ is an isomorphism. Of course, $\mathrm{Ad} u$ also defines an isomorphism of $M\left(A \times{ }_{\delta} G\right)$ onto $M\left(A \times{ }_{\gamma} G\right)$ such that $\operatorname{Ad} u(\delta(a))=\gamma(a)$ for each $a \in A$. Let $\phi:\left(A \times{ }_{\gamma} G\right)^{\wedge} \rightarrow$ $\left(A \times{ }_{\delta} G\right)^{\wedge}$ denote the induced homeomorphism; i.e. $\Phi([\nu])=[\nu \circ \operatorname{Ad} u]$. Now, $p:\left(A \times{ }_{\gamma} G\right)^{\wedge} \rightarrow \hat{A}$ is the map $[\nu] \mapsto\left[\nu^{-} \circ \gamma\right]$ and $q:\left(A \times{ }_{\delta} G\right)^{\wedge} \mapsto \hat{A}$ is the map $[\rho] \rightarrow\left[\rho^{-} \circ \delta\right]$. Since $\operatorname{Ad} u \circ \delta=\gamma$, the diagram

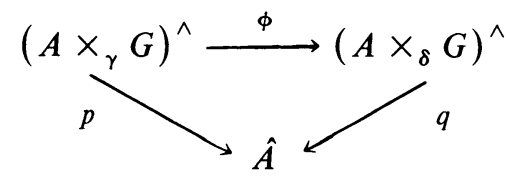

commutes.

Thus, it suffices to see that $\phi$ is $G$-equivariant. But, the dual action of $G$ on either algebra is given for $s \in G$ by $\hat{\delta}_{s}=\operatorname{Ad}(1 \otimes \rho(s))$. Now, $1 \otimes \rho(s)$ commutes with $A \otimes C_{r}^{*}(G)$ and hence with $u \in M\left(A \otimes C_{r}^{*}(G)\right)$. Therefore,

$$
\begin{aligned}
\phi(s \cdot[\nu]) & =\phi\left[\nu \circ \hat{\delta}_{s^{-1}}\right]=\left[\nu \circ \hat{\delta}_{s^{-1}} \circ \operatorname{Ad} u\right]=\left[\nu \circ \operatorname{Ad} u \circ \hat{\delta}_{s^{-1}}\right] \\
& =s \cdot[\nu \circ \operatorname{Ad} u]=s \cdot \phi[\nu]
\end{aligned}
$$

as required.

To see the converse, we suppose that $\phi:\left(A \times_{\gamma} G\right)^{\wedge} \rightarrow\left(A \times_{\delta} G\right)^{\wedge}$ is a $G$-bundle isomorphism over $\hat{A}$. Since $\delta$ and $\gamma$ are locally unitary, we can find an open cover $\left\{N_{i}\right\}$ of $\hat{A}$ and elements $\tilde{u}_{i}, \tilde{v}_{i}$ in $M\left(A / I_{i} \otimes C_{r}^{*}(G)\right)$, where $I_{i}=\bigcap\left\{\operatorname{ker} \pi \mid \pi \in N_{i}\right\}$ so that

$$
\delta^{I_{i}}\left(a+I_{i}\right)=\tilde{u}_{i}\left(\left(a+I_{i}\right) \otimes 1\right) \tilde{u}_{i}^{*} \quad \text { and } \quad \gamma^{I_{i}}\left(a+I_{i}\right)=\tilde{v}_{i}\left(\left(a+I_{i}\right) \otimes 1\right) \tilde{v}_{i}^{*} .
$$


If we let $\eta_{i}: A \rightarrow A / I_{i}$ be the natural projection, and let $u_{i}, v_{i}$ be elements of $M\left(A \otimes C_{r}^{*}(G)\right)$ with $\left(\eta_{i} \otimes i\right)^{-}\left(u_{i}\right)=\tilde{u}_{i}$ and $\left(\eta_{i} \otimes i\right)^{-}\left(v_{i}\right)=\tilde{v}_{i}$, then these equations become

$$
\left(\eta_{i} \otimes i\right)^{-}(\delta(a))=\left(\eta_{i} \otimes i\right)^{-}\left(u_{i}(a \otimes 1) u_{i}^{*}\right)
$$

and

$$
\left(\eta_{i} \otimes i\right)^{-}(\gamma(a))=\left(\eta_{i} \otimes i\right)^{-}\left(v_{i}(a \otimes 1) v_{i}^{*}\right)
$$

for all $a \in A$. In fact, if $a$ is supported on $N_{i}$, then since $I_{i}$ is invariant for $\delta$ and $\gamma$ the equations become $\delta(a)=u_{i}(a \otimes 1) u_{i}^{*}$ and $\gamma(a)=v_{i}(a \otimes 1) v_{i}^{*}$.

Now, we concentrate on $\delta$ for the moment and use Theorem 5.9 to get two trivializations of $p^{-1}\left(N_{i} \cap N_{j}\right)=p^{-1}\left(N_{i j}\right)$ :

$$
\begin{aligned}
N_{i j} \times G \rightarrow & p^{-1}\left(N_{i j}\right) \leftarrow N_{i j} \times G \\
& \searrow \downarrow \prec \\
& N_{i j}
\end{aligned}
$$

where the two maps $N_{i j} \times G \rightarrow p^{-1}\left(N_{i j}\right)$ are given by

$$
(\pi, s) \mapsto\left[\pi \times(\pi \otimes i)^{-}\left(u_{i}\right)\right] \circ \hat{\delta}_{s} \quad \text { and } \quad(\pi, t) \mapsto\left[\pi \times(\pi \otimes i)^{-}\left(u_{j}\right)\right] \circ \hat{\delta}_{t} .
$$

Since these maps are $G$-equivariant, the composition of one with the inverse of the other is of the form $(\pi, s) \mapsto\left(\pi, \gamma_{i j}(\pi) s\right): N_{i j} \times G \rightarrow N_{i j} \times G$, where $\gamma_{i j}: N_{i j} \rightarrow G$ is continuous. That is, for $\pi \in N_{i j}$

$$
\left[\pi \times(\pi \otimes i)^{-}\left(u_{j}\right)\right] \circ \hat{\delta}_{s}=\left[\pi \times(\pi \otimes i)^{-}\left(u_{i}\right)\right] \circ \hat{\delta}_{\left(\gamma_{i j}(\pi) s\right)} .
$$

Equivalently, we get for $\pi \in N_{i j}$

$$
\left[\pi \times(\pi \otimes i)^{-}\left(u_{j}\right)\right]=\left[\pi \times(\pi \otimes i)^{-}\left(u_{i}\right)\right] \circ \hat{\delta}_{\gamma_{1}(\pi)} .
$$

If we apply these representations to the generators of $A \times_{\delta} G$ and use the fact that $\pi$ is irreducible we get that $S_{f \cdot \gamma_{t}(\pi)}\left((\pi \otimes i)^{-}\left(u_{i}\right)\right)=S_{f}\left((\pi \otimes i)^{-}\left(u_{j}\right)\right)$ for $\pi \in N_{i j}$. By a straightforward calculation the left-hand side equals

$$
S_{f}\left[(\pi \otimes i)^{-}\left(u_{i}\right)\left(1 \otimes \lambda\left(\gamma_{i j}(\pi)\right)\right)\right] .
$$

Since this holds for all $f \in A(G)$, we get the equation

$$
(\pi \otimes i)^{-}\left(u_{i}\right)\left(1 \otimes \lambda\left(\gamma_{i j}(\pi)\right)\right)=(\pi \otimes i)^{-}\left(u_{j}\right) \text { for all } \pi \in N_{i j} .
$$

Similar considerations show that we have transition functions $\left\{\chi_{i j}\right\}$ for the bundle $q:\left(A \times{ }_{\gamma} G\right)^{\wedge} \rightarrow \hat{A}$ so that

$$
(\pi \otimes i)^{-}\left(v_{i}\right)\left(1 \otimes \lambda\left(\chi_{i j}(\pi)\right)\right)=(\pi \otimes i)^{-}\left(v_{j}\right)
$$

for all $\pi \in N_{i j}$.

Since the two bundles are $G$-isomorphic, we can assume (by shrinking the $N_{i}$ 's if necessary) that there are continuous functions $\beta_{i}: N_{i}^{-} \rightarrow G$ so that $\chi_{i j}=\beta_{i}^{-1} \gamma_{i j} \beta_{j}$ on $N_{i j}$. Now, $\pi \mapsto \lambda\left(\beta_{i}(\pi)\right)$ is a strictly continuous bounded function from $\bar{N}_{i}$ into $M\left(C_{r}^{*}(G)\right)$ and hence defines a multiplier of $C_{0}\left(\bar{N}_{i}, C_{r}^{*}(G)\right)$. Since $\bar{N}_{i}=\left(A / I_{i}\right)^{\wedge}$, 
the usual factorization trick shows that the multipliers of $C_{0}\left(\bar{N}_{i}\right) \otimes C_{r}^{*}(G)$ are naturally embedded in $M\left(A / I_{i} \otimes C_{r}^{*}(G)\right)$. Thus, $\pi \rightarrow \lambda\left(\beta_{i}(\pi)\right)$ is an element of $M\left(A / I_{i} \otimes C_{r}^{*}(G)\right)$ which in the representation $(\pi \otimes i)^{-}$is given by $1 \otimes \lambda\left(\beta_{i}(\pi)\right)$. Now, if we replace $\tilde{u}_{i}$ in $M\left(A / I_{i} \otimes C_{r}^{*}(G)\right)$ by $\tilde{u}_{i}\left(1 \otimes \lambda \circ \beta_{i}\right)$, then we can assume that the $u_{i}$ 's transform as follows:

$$
(\pi \otimes i)^{-}\left(u_{i}\right)\left(1 \otimes \lambda\left(\chi_{i j}(\pi)\right)\right)=(\pi \otimes i)^{-}\left(u_{j}\right) .
$$

Moreover, these new $u_{i}$ 's still implement $\delta$ locally. Now, let $W_{i}=v_{i} u_{i}^{*}$. Then, for $\pi \in N_{i j}$ we see that $(\pi \otimes i)^{-}\left(W_{i}\right)=(\pi \otimes i)^{-}\left(W_{j}\right)$. Since $\hat{A}$ is Hausdorff, any element of $A \otimes C_{r}^{*}(G)$ can be approximated by a finite sum of the form $\sum a_{i} \otimes x_{i}$, where $a_{i}$ is supported on $N_{i}$. Hence, we can define a multiplier, $W$, of $A \otimes C_{r}^{*}(G)$ by

$$
W \cdot\left(\sum a_{i} \otimes x_{i}\right)=\sum W_{i}\left(a_{i} \otimes x_{i}\right) \quad \text { and } \quad\left(\sum a_{i} \otimes x_{i}\right) \cdot W=\sum\left(a_{i} \otimes x_{i}\right) W_{i} .
$$

This is well defined since $(\pi \otimes i)^{-}\left(W_{i}\right)=(\pi \otimes i)^{-}\left(W_{j}\right)$ for all $\pi \in N_{i j}$.

The element $W$ is clearly unitary and satisfies $\operatorname{Ad} W \circ \delta=\gamma$. Since $\gamma$ is nondegenerate, $\left(1 \otimes C_{r}^{*}(G)\right) \gamma(A)$ is dense in $A \otimes C_{r}^{*}(G)$ by [12, Theorem 5] and so

$$
\left(1 \otimes C_{r}^{*}(G)\right) \gamma(A) \cdot W=\left(1 \otimes C_{r}^{*}(G)\right) W \delta(A)
$$

is dense in $A \otimes C_{r}^{*}(G)$. Hence, $W$ satisfies (2) and (3) of the definition of a $\delta$-one cocycle. Now, a straightforward calculation shows that for any $\pi \in \hat{A}$

$$
(\pi \otimes i \otimes i)^{-}\left(i \otimes \delta_{G}\right)^{-}(W)=(\pi \otimes i \otimes i)^{-}[(W \otimes 1)(\delta \otimes i)(W)]
$$

and so $W$ also satisfies condition (1). Therefore, $\gamma$ and $\delta$ are exterior equivalent, and the theorem is proved.

5.12. Remark. It is easy to see that if two nondegenerate coactions are exterior equivalent and one of them is locally unitary, then so is the other. As we shall see in $\S 6$ this will show that for a stable $C^{*}$-algebra with paracompact Hausdorff spectrum, $T$, and a separable locally compact group, $G$, the exterior equivalence classes of locally unitary coactions of $G$ are in bijective correspondence with the isomorphism classes of locally trivial principal $G$-bundles over $T$.

Our main examples of pointwise and locally unitary coactions will be dual coactions $\hat{\alpha}$ on reduced crossed products $A \times_{\alpha, r} G$. Roughly speaking, $\hat{\alpha}$ will be pointwise unitary on $A \times_{\alpha, r} G$ if $\hat{A}$ is a (Cartan) principal $G$-bundle, and locally unitary if $\hat{A}$ is a locally trivial $G$-bundle. The abelian version of these results is in [20, Lemma 2.4].

5.13. Proposition. Suppose $A$ is a $C^{*}$-algebra with Hausdorff spectrum and $\alpha: G \rightarrow$ Aut $A$ is an automorphism group such that $\hat{A}$ is a (Cartan) principal G-bundle. Then $A \times{ }_{\alpha} G=A \times{ }_{\alpha, r} G$ has Hausdorff spectrum $\hat{A} / G$ and the dual coaction of $G$ on $A \times{ }_{\alpha} G$ is pointwise unitary.

Proof. First we observe that Green's version of the Mackey machine $[7,8]$ shows that $\pi \rightarrow$ Ind $\pi$ induces a homeomorphism of $\hat{A} / G$ onto $\left(A \times{ }_{\alpha} G\right)^{\wedge}[\mathbf{2 0}$, Theorem 1.1(1)], and since $\oplus \operatorname{Ind} \pi=\operatorname{Ind}(\oplus \pi)$ is therefore faithful, the reduced crossed product is all of $A \times_{\alpha} G$ [16, Theorem 7.7.5]. Now, recall from Example 2.3(1) that 
if $\nu: A \rightarrow \mathscr{B}(H)$ is faithful, then the dual coaction $\delta$ is defined for $x \in A \times{ }_{\alpha} G$ by

$$
((\operatorname{Ind} \nu) \otimes i)(\delta(x))=\left(1 \otimes W_{G}\right)(\text { Ind } \pi(x) \otimes 1)\left(1 \otimes W_{G}^{*}\right) .
$$

Let $i_{A}, 1 \otimes \lambda$ denote the embeddings of $A, C_{r}^{*}(G)$ in $M\left(A \times{ }_{\alpha} G\right)$. Then it is easy to check that

$$
\delta^{-}\left(i_{A}(a)\right)=i_{A}(a) \otimes 1 \quad \text { and } \delta^{-}(1 \otimes \lambda(g))=1 \otimes \delta_{G}(\lambda(g))
$$

in $M\left(\left(A \times_{\alpha} G\right) \otimes C_{r}^{*}(G)\right)$. The same calculations then show that $(*)$ holds for any representation $\nu$ of $A$. Thus, it will be enough to prove $1 \otimes W_{G}$ belongs to $M\left(\right.$ Ind $\left.\pi\left(A \times_{\alpha} G\right) \otimes C_{r}^{*}(G)\right)$ for every $\pi \in \hat{A}$. However, $A \times_{\alpha} G$ has Hausdorff spectrum, so

$$
\text { Ind } \pi\left(A \times_{\alpha} G\right)=K\left(H_{\text {Ind } \pi}\right)=K\left(H_{\pi} \otimes L^{2}(G)\right)=K\left(H_{\pi}\right) \otimes K\left(L^{2}(G)\right) .
$$

We already know that $W_{G} \in M\left(K\left(L^{2}(G)\right) \otimes C_{r}^{*}(G)\right)$ by Example 2.3(4), so that $1 \otimes W_{G}$ implements $\delta$ in the representation Ind $\pi$ and we are done.

5.14. Proposition. Let $A$ be a $C^{*}$-algebra with Hausdorff spectrum and suppose $\alpha: G \rightarrow$ Aut $A$ is a locally compact automorphism group such that $\hat{A}$ with the action of $G$ is a locally trivial principal $G$-bundle over a Hausdorff space $X$. Then, the dual coaction of $G$ on $A \times{ }_{\alpha} G=A \times{ }_{\alpha, r} G$ is locally unitary.

The idea of the proof is to use the ideal theory of $\$ 4$ to reduce to the case where $\hat{A}=X \times G$, and calculate the crossed product explicitly in that case. To do this, we want to use some simple properties of continuous fields of $C^{*}$-algebras [3, Chapter $10]$. Let $E$ be a continuous field of elementary $C^{*}$-algebras over a locally compact Hausdorff space $X$, and let $H$ be a fixed Hilbert space. Then, there is a continuous field $E \otimes K(H)$ whose fiber at $x$ is $E_{x} \otimes K(H)$ and whose section algebra is $\Gamma_{0}(E) \otimes K(H)$ (take, as continuous sections, those which are locally uniformly approximable by $\sum_{i} f_{i} \otimes k_{i}$ for $\left.f_{i} \in \Gamma_{0}(E), k_{i} \in K(H)\right)$. We pause to prove

5.15. LEMMA. Let $A$ be a $C^{*}$-algebra with Hausdorff spectrum $X \times G$, and suppose $\alpha: G \rightarrow$ Aut $A$ induces the left action of $G$ on $X \times G$ (i.e., $g(x, h)=(x, g h)$ ). Realizing $A$ as the section algebra $\Gamma_{0}(E)$ of a continuous field $E$ of elementary $C^{*}$-algebras over $X \times G$, let $\pi(x, t)$ denote the representation $a \mapsto a(x, t)$ of $A$. Then the map $\Phi$ defined by

$$
\Phi(b)(x)=\operatorname{Ind} \pi(x, e)(b)
$$

is an isomorphism of $A \times_{\alpha} G$ onto $\Gamma_{0}\left(\left.E\right|_{X \times\{e\}} \otimes K\left(L^{2}(G)\right)\right)$.

Proof. By Proposition 5.13 and its proof, Ind $\pi(x, e)$ is irreducible on $A \times{ }_{\alpha} G=$ $A \times_{\alpha, r} G$ and its range is the compact operators on $H_{(x, e)} \otimes L^{2}(G)$, that is, $E_{(x, e)} \otimes K\left(L^{2}(G)\right)$. The map $\Phi$ preserves the natural actions of $C_{b}(X)$ (and so $\left.C_{0}(X)\right)$ : hence the result will follow if we can show that $\Phi(b)$ is a continuous section of $\left.E\right|_{X \times\{e\}} \otimes K\left(L^{2}(G)\right)$. To this end let $b=i_{A}(a)(1 \otimes \lambda(g))$, where $a \in \Gamma_{c}(E) \subseteq A$ and $g \in C_{c}(G)$. Then, we can view Ind $\pi(x, e)\left(i_{A}(a)\right)$ as the continuous function $s \mapsto \pi(x, e)\left(\alpha_{s}^{-1}(a)\right)$ in $C_{b}\left(G, E_{(x, e)}\right)$. Since $\pi(x, e) \circ \alpha_{s}^{-1}$ is equivalent to $\pi(x, s)$ by our hypothesis on $\alpha$, and since $a$ has compact support, this map has compact 
support in $G$. In fact, we may assume that there are compact sets $C \subseteq X$ and $L \subseteq G$ with $\pi(x, e)\left(\alpha_{s}^{-1}(a)\right)=0$ if $(x, s) \notin C \times L$. Thus, we can approximate $s \mapsto \alpha_{s}^{-1}(a)$ uniformly by an element of the form $\sum a_{i} \otimes f_{i}$, where $a_{i} \in A$ and $f_{i} \in C_{c}(G)$. Then, Ind $\pi(x, e)(b)$ is uniformly approximated on $C \times L$ by

$$
\sum \pi(x, e)\left(a_{i}\right) \otimes M_{f_{i}} \lambda(g) \in E_{(x, e)} \otimes K\left(L^{2}(G)\right) .
$$

Thus, $x \mapsto \operatorname{Ind} \pi(x, e)(b)$ is a continuous section of $\left.E\right|_{X \times\{e\}} \otimes K\left(L^{2}(G)\right)$ and the result follows.

Proof of Proposition 5.14. We first consider the case where $\hat{A}$ is trivial. So, suppose $\hat{A}=X \times G$, and $\alpha$ induces the canonical left action of $G$ on $\hat{A}$ (we will use the notation of the lemma). Recall from the proof of Proposition 5.13 that, if $\delta$ is the dual coaction, then for any nondegenerate representation $\nu$ of $A$ we have for $b \in A \times{ }_{\alpha} G$,

$$
((\operatorname{Ind} \nu) \otimes i)^{-}(\delta(b))=\left(1 \otimes W_{G}\right)(\operatorname{Ind} \nu(b) \otimes 1)\left(1 \otimes W_{G}^{*}\right) .
$$

If we take $\nu=\oplus_{x \in X} \pi(x, e)$, then Ind $\nu \cong \oplus_{x \in X}$ Ind $\pi(x, e)$ is faithful and acts in $\left(\oplus_{x \in X} H_{(x, e)}\right) \otimes L^{2}(G)$. Moreover, the following diagram commutes:

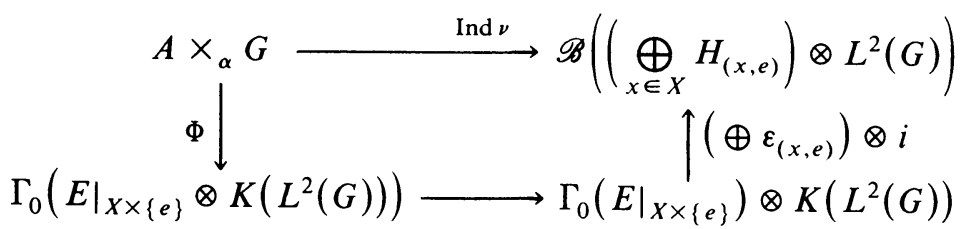

where $\varepsilon_{(x, e)}$ denotes evaluation at $(x, e)$. Now, the unitary $1 \otimes W_{G}$ on $\left(\oplus_{x \in X} H_{(x, e)}\right) \otimes L^{2}(G) \otimes L^{2}(G)$ is the image of

$$
1 \otimes W_{G} \in M\left(\Gamma_{0}\left(\left.E\right|_{X \times\{e\}}\right) \otimes K\left(L^{2}(G)\right) \otimes C_{r}^{*}(G)\right)
$$

under the map $\left(\oplus_{x \in X} \varepsilon_{(x, e)}\right) \otimes i \otimes i$. Thus, pulling $1 \otimes W_{G}$ back to

$$
M\left(\left(A \times_{\alpha} G\right) \otimes C_{r}^{*}(G)\right)
$$

along the lower route shows that $\delta$ is unitary.

Now, suppose $\hat{A}$ is a locally trivial $G$-bundle and write $p: \hat{A} \rightarrow X$ for the orbit map. By Proposition $5.13\left(A \times{ }_{\alpha} G\right)^{\wedge}$ is homeomorphic to $X$, and $\delta$ is pointwise unitary. Given $x_{0} \in X$, choose a compact neighborhood $N$ of $x_{0}$ such that $p^{-1}(N)$ $\cong N \times G$ and let $I=\bigcap\{\operatorname{ker} \pi \mid p(\pi) \in N\}$. Then $I$ is invariant under $\alpha$ and

$$
I \times{ }_{\alpha} G=\bigcap\{\operatorname{ker}(\operatorname{Ind} \pi) \mid p(\pi) \in N\}
$$

so that $J=I \times{ }_{\alpha} G$ is invariant under $\delta$ and $\delta^{J}$ is a nondegenerate coaction by Proposition 5.3(2). It will be enough for us to prove that $\delta^{J}$ is unitary. However, a routine check shows that if $\alpha^{I}$ is the induced action of $G$ on $A / I$ and $\theta$ is the canonical isomorphism of $\left(A \times{ }_{\alpha} G\right) /\left(I \times{ }_{\alpha} G\right)$ onto $(A / I) \times{ }_{\alpha^{\prime}} G$, then we have a commutative diagram:

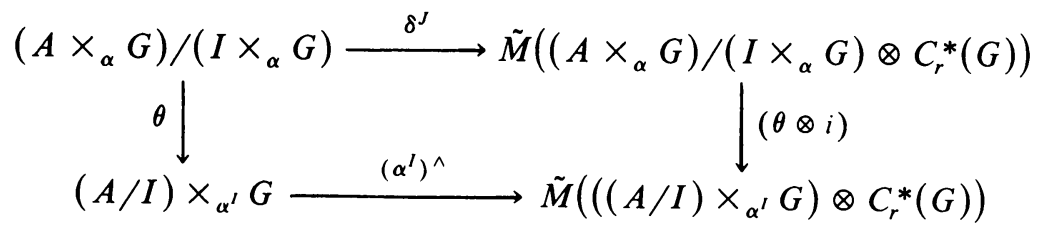


where $\left(\alpha^{I}\right)^{\wedge}$ denotes the coaction dual to $\alpha^{I}$. Because $(A / I)^{\wedge}=p^{-1}(N)$ is a trivial $G$-bundle for the action $\alpha^{I}$ induced by $\alpha$, we have already seen that $\left(\alpha^{I}\right)^{\wedge}$ is unitary. Therefore, $\delta^{J}$ is also unitary and so $\delta$ is locally unitary.

6. The construction of crossed products with given spectrum. We have seen that the spectrum of the crossed product $A \times{ }_{\delta} G$ by a locally unitary coaction is in a natural way a locally trivial $G$-bundle over $\hat{A}$. We shall now show how to construct coactions $\delta$ so that $p:\left(A \times{ }_{\delta} G\right)^{\wedge} \rightarrow \hat{A}$ is a given (Cartan) principal $G$-bundle.

The idea of our construction is simple. Given a (Cartan) principal $G$-bundle $q: E \rightarrow T=E / G$, let $\delta$ be the dual coaction of $G$ on the transformation group $C^{*}$-algebra $C^{*}(G, E)=C_{0}(E) \times{ }_{\alpha} G$. By 5.13 and 5.14 , this algebra has spectrum $T$, and $\delta$ is pointwise unitary; locally unitary if $q: E \rightarrow T$ is locally trivial. By the duality theory of $[10], C^{*}(G, E) \times{ }_{\delta} G$ is isomorphic to $C_{0}(E) \otimes K\left(L^{2}(G)\right)$ and hence has spectrum $E$. Theorem 5.5 then gives a map $p: E \rightarrow T$ which (up to the suppressed identifications) is just $q: E \rightarrow T$. On the other hand, a theorem of Green [7] asserts that if $q: E \rightarrow T$ is locally trivial, then $C^{*}(G, E) \cong C_{0}\left(T, K\left(L^{2}(G)\right)\right)$ and so we will have constructed a coaction of $G$ on $C_{0}(T, K)=A$ so that $p:\left(A \times{ }_{\delta} G\right)^{\wedge}$ $\rightarrow \hat{A}=T$ is isomorphic to $q: E \rightarrow T$.

In making this argument precise we need properties of the duality isomorphism which are not made explicit in [10]. In particular, we need the explicit realization of the dual action of $G$ on $B \otimes K\left(L^{2}(G)\right) \cong\left(B \times{ }_{\alpha, r} G\right) \times_{\delta} G$ which was not given in [10].

To this end, let $\alpha: G \rightarrow$ Aut $B$ be an automorphism group and let $\delta$ be the dual coaction of $G$ on $B \times{ }_{\alpha, r} G$. Let $\tau$ denote the left regular representation of $G$ on $C_{0}(G)$. Let $\pi$ be a faithful representation of $B$ on $H$ so that Ind $\pi$ is a faithful representation of $B \times{ }_{\alpha, r} G$ on $H \otimes L^{2}(G)$. Thus, by 2.3(1) we have a faithful representation of $\left(B \times_{\alpha, r} G\right) \times{ }_{\delta} G$ on $\left(H \otimes L^{2}(G)\right) \otimes L^{2}(G)$ given by

$$
\delta(x)\left(1 \otimes M_{f}\right) \rightarrow\left(1 \otimes W_{G}\right)(\text { Ind } \pi(x) \otimes 1)\left(1 \otimes W_{G}^{*}\right)\left(1 \otimes 1 \otimes M_{f}\right) .
$$

Moreover, $\operatorname{Ind}(\pi \otimes M)$ is a faithful representation of $\left(B \otimes C_{0}(G)\right) \times_{\alpha \otimes \tau, r} G$ on $\left(H \otimes L^{2}(G)\right) \otimes L^{2}(G)$. With these concrete realizations we have the following result [10, Proposition 3.1].

6.1. LeMma. Let $(B, G, \alpha)$ be as above and let

$$
\left(B \times_{\alpha, r} G\right) \times_{\delta} G,\left(B \otimes C_{0}(G)\right) \times_{\alpha \otimes \tau, r} G
$$

be realized on $\left(H \otimes L^{2}(G)\right) \otimes L^{2}(G)$ as above. If $\Sigma$ denotes the unitary on this space which switches the last two variables, then $\operatorname{Ad}\left(\Sigma\left(1 \otimes W_{G}^{*}\right)\right)$ is an isomorphism of $\left(B \times_{\alpha, r} G\right) \times_{\delta} G$ onto $\left(B \otimes C_{0}(G)\right) \times_{\alpha \otimes \tau, r} G$ which takes the dual action $\hat{\delta}_{s}=$ $\operatorname{Ad}(1 \otimes 1 \otimes \rho(s))$ to $\operatorname{Ad}(1 \otimes \rho(s) \otimes 1)$, which is the canonical extension of the right translation, $1 \otimes \sigma_{s}$ on $B \otimes C_{0}(G)$, to the crossed product, $\left(B \otimes C_{0}(G)\right) \times_{\alpha \otimes \tau, r} G$.

Proof. By a straightforward calculation, we see that $\Phi=\operatorname{Ad}\left(\Sigma\left(1 \otimes W_{G}^{*}\right)\right)$ satisfies:

$$
\begin{array}{ll}
\Phi(\bar{\delta}(1 \otimes \lambda(g)))=1 \otimes 1 \otimes \lambda(g), & \lambda(g) \in C_{r}^{*}(G) ; \\
\Phi(\bar{\delta}(\tilde{\pi}(b)))=(\pi \otimes M)^{\sim}(b \otimes 1), & b \in B ; \\
\Phi\left(1 \otimes 1 \otimes M_{f}\right)=(\pi \otimes M)^{\sim}(1 \otimes f), & f \in C_{0}(G)
\end{array}
$$


where Ind $\pi=\tilde{\pi} \times(1 \otimes \lambda)$ and $\operatorname{Ind}(\pi \otimes M)=(\pi \otimes M)^{\sim} \times(1 \otimes 1 \otimes \lambda)$. As products of elements on the left-hand side generate $\left(B \times_{\alpha, r} G\right) \times_{\delta} G$ and products of elements on the right-hand side generate $\left(B \otimes C_{0}(G)\right) \times_{\alpha \otimes \tau, r} G, \Phi$ is an isomorphism as claimed. Another calculation yields $\Phi(1 \otimes 1 \otimes \rho(s))=1 \otimes \rho(s) \otimes 1$ so that the last statement of the lemma is clear.

6.2. Lemma. Let $\Psi \in \operatorname{Aut}\left(B \otimes C_{0}(G)\right)$ be defined by $\Psi(f)(s)=\alpha_{s^{-1}}(f(s))$, where $(B, G, \alpha)$ is as above. Then,

$\Psi \circ\left(\alpha_{s} \otimes \tau_{s}\right) \circ \Psi^{-1}=1 \otimes \tau_{s} \quad$ and $\quad \Psi \circ\left(1 \otimes \sigma_{s}\right) \circ \Psi^{-1}=\alpha_{s} \otimes \sigma_{s} \quad$ for $s \in G$.

Thus, $\Psi$ induces an isomorphism:

$$
\begin{aligned}
\bar{\Psi}:(B \otimes & \left.C_{0}(G)\right) \times_{\alpha \otimes \tau, r} G \rightarrow\left(B \otimes C_{0}(G)\right) \times_{1 \otimes \tau, r} G \\
& =B \otimes\left(C_{0}(G) \times_{\tau, r} G\right) \\
& \cong B \otimes K\left(L^{2}(G)\right)
\end{aligned}
$$

which carries the canonical extension of $1 \otimes \sigma_{s}$ to the canonical extension of $\alpha_{s} \otimes \sigma_{s}$.

Proof. The two equations are straightforward calculations and they immediately imply the second statement.

So the refined version of [10, Theorem 3.6] that we need is the following

6.3. TheOREM. Let $\alpha: G \rightarrow$ Aut $B$ be an automorphism group and let $\delta$ be the dual coaction of $G$ on $B \times_{\alpha, r} G$. Then there is an isomorphism $\phi:\left(B \times_{\alpha, r} G\right) \times_{\delta} G \rightarrow B \otimes$ $K\left(L^{2}(G)\right)$ which carries the dual action $\hat{\delta}$ to $\alpha \otimes \sigma$. That is, $\phi \circ \hat{\delta}_{s}{ }^{\circ} \phi^{-1}=\alpha_{s} \otimes \sigma_{s}$ for all $s \in G$.

Proof. $\bar{\Psi} \circ \Phi$ is the desired isomorphism.

6.4. Corollary. The canonical homeomorphism $\hat{B} \rightarrow(B \otimes K)^{\wedge}$ intertwines the induced actions $\left(\alpha_{s}\right)^{*}$ and $\left(\phi \circ \hat{\delta}_{s} \circ \phi^{-1}\right)^{*}$.

Proof. $\left(\phi \circ \hat{\delta}_{s} \circ \phi^{-1}\right)^{*}=\left(\alpha_{s} \otimes \sigma_{s}\right)^{*}=\left(\alpha_{s}\right)^{*} \times i$.

6.5. TheOREM. Let $q$ : $E \rightarrow T=E / G$ be a (Cartan) principal $G$-bundle and let $\alpha$ : $G \rightarrow \operatorname{Aut}\left(C_{0}(E)\right)$ be the induced action. Let $A=C^{*}(G, E)=C_{0}(E) \times_{\alpha, r} G$ and let $\delta$ be the dual coaction of $G$ on $A$. Let $\phi: A \times{ }_{\delta} G \rightarrow C_{0}(E) \otimes K\left(L^{2}(G)\right)$ be the isomorphism of Theorem 6.3. Then the following diagram commutes and is an isomorphism of G-bundles:

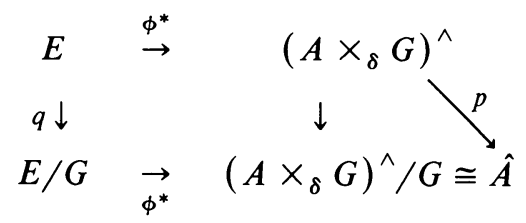

Proof. By the corollary, $\phi^{*}$ is equivariant and so induces the isomorphism of principal $G$-bundles given by the left-hand square. That the right-hand triangle commutes follows from 5.5(2), (3). So it suffices to see that the continuous bijection $\left(A \times{ }_{\delta} G\right)^{\wedge} / G \rightarrow \hat{A}$ is a homeomorphism. However, the composition $E / G \rightarrow$ $\left(A \times{ }_{\delta} G\right)^{\wedge} / G \rightarrow \hat{A}$ is just the homeomorphism defined in [18, Lemma 3.3]. Thus, $\left(A \times{ }_{\delta} G\right)^{\wedge} / G \rightarrow \hat{A}$ is a homeomorphism and we are done. 
6.6. Corollary. Let $K$ denote the compact operators on a separable Hilbert space. Let $G$ be a separable locally compact group and let $q: E \rightarrow T$ be a (Cartan) principal $G$-bundle over the locally compact, paracompact space $T$. Then there is a pointwise unitary coaction $\delta$ of $G$ on $C_{0}(T, K)=A$ so that $p:\left(A \times{ }_{\delta} G\right)^{\wedge} \rightarrow \hat{A}=T$ is isomorphic to $q: E \rightarrow T$. Moreover, if $q: E \rightarrow T$ is locally trivial, then $\delta$ can be chosen to be locally unitary.

Proof. By Green's result [7, Theorem 14], $B=C^{*}(G, E)$ is a continuous trace $C^{*}$-algebra with spectrum $T$ and Dixmier-Douady invariant 0 . Thus, by Dixmier's result [17, Corollary 1.5], $K \otimes B \cong C_{0}(T, K)$ and so if $\delta_{1}$ is the pointwise unitary coaction of $G$ on $B$ so that $p:\left(B \times_{\delta_{1}} G\right)^{\wedge} \rightarrow \hat{B} \cong T$ is isomorphic to $q: E \rightarrow T$, then $\delta=i \otimes \delta$ is clearly a pointwise unitary coaction of $K \otimes B$ and the following diagram is an isomorphism of $G$-bundles:

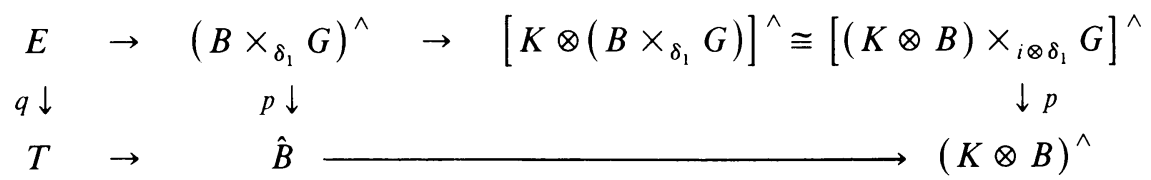

Since $K \otimes B \cong C_{0}(T, K)$ we are finished except for observing that $\delta_{1}$ and hence $i \otimes \delta_{1}=\delta$ may be taken to be locally unitary if $q: E \rightarrow T$ is locally trivial by Proposition 5.14 and Theorem 6.5.

6.7. TheOREM. Let $A$ be a stable $C^{*}$-algebra with paracompact, Hausdorff spectrum, $T$. Let $q: E \rightarrow T$ be a (Cartan) principal $G$-bundle over $T$ where $G$ is a separable locally compact group. Then there is a pointwise unitary coaction $\delta$ of $G$ on $A$ so that $p:\left(A \times{ }_{\delta} G\right)^{\wedge} \rightarrow \hat{A}=T$ is isomorphic to $q: E \rightarrow T$. If $q: E \rightarrow T$ is locally trivial, then $\delta$ may be chosen to be locally unitary.

Proof. By 4.3 and 3.9 of [18] there is an isomorphism $\phi: A \rightarrow A \otimes_{C_{0}(T)} C_{0}(T, K)$ so that on spectra, $\hat{\phi}: T=\Delta(T \times T) \rightarrow \hat{A}=T$ is the identity mapping. Here, $A \otimes{ }_{C_{0}(T)} C_{0}(T, K)$ is the quotient of $A \otimes C_{0}(T, K)$ by the ideal $I$ generated by $a f \otimes b-a \otimes f b$, where $a \in A, f \in C_{0}(T)$, and $b \in C_{0}(T, K)$, and $\Delta(T \times T)=$ $\{(x, x) \mid x \in T\}$. Let $\delta$ be a pointwise unitary coaction (locally unitary if $q: E \rightarrow T$ is locally trivial) of $C_{0}(T, K)$ so that $p:\left(C_{0}(T, K) \times_{\delta} G\right)^{\wedge} \rightarrow \hat{A}=T$ is isomorphic to $q: E \rightarrow T$. Then $i \otimes \delta$ defines a coaction of $A \otimes C_{0}(T, K)$ which preserves the $C_{0}(T)$-module structure and hence satisfies the condition on approximate identities with regard to the ideal $I$. By Remark 4.10, $(i \otimes \delta)^{I}$ is a nondegenerate coaction of $A \otimes{ }_{C_{0}(T)} C_{0}(T, K)$. We will be sloppy and denote this coaction by $i \otimes \delta$. It is easy to see that $i \otimes \delta$ is pointwise unitary (or locally unitary if $\delta$ is) and that there is a natural isomorphism $\left(A \otimes_{C_{0}(T)} C_{0}(T, K)\right) \times_{i \otimes \delta} G \cong A \otimes_{C_{0}(T)}\left(C_{0}(T, K) \times_{\delta} G\right)$ yielding the commutative diagram:

$$
\begin{array}{rlr}
{\left[\left(A \otimes_{C_{0}(T)} C_{0}(T, K)\right) \times_{i \otimes \delta} G\right]^{\wedge}} & \cong & {\left[A \otimes_{C_{0}(T)}\left(C_{0}(T, K) \times_{\delta} G\right)\right]^{\wedge}} \\
p \downarrow & \downarrow \Delta(i \times p) \\
{\left[A \otimes_{C_{0}(T)} C_{0}(T, K)\right]^{\wedge}} & \cong & \Delta\left(\hat{A} \times C_{0}(T, K)^{\wedge}\right)
\end{array}
$$


But, $\quad \Delta\left(\hat{A} \times C_{0}(T, K)^{\wedge}\right)=\Delta(T \times T)=T$ and $\left[A \otimes_{C_{0}(T)} C_{0}(T, K) \times_{\delta} G\right]^{\wedge} \cong$ $\left(C_{0}(T, K) \times{ }_{\delta} G\right)^{\wedge}$, and the diagram

$$
\begin{array}{cccc}
{\left[A \otimes_{C_{0}(T)}\left(C_{0}(T, K) \times_{\delta} G\right)\right]^{\wedge}} & \cong & \left(C_{0}(T, K) \times_{\delta} G\right)^{\wedge} & \cong E \\
\downarrow & & \downarrow p & \downarrow q \\
\Delta\left(\hat{A} \times C_{0}(T, K)^{\wedge}\right) & \cong & T=C_{0}(T, K)^{\wedge} & =T
\end{array}
$$

commutes. As the isomorphism $\phi: A \rightarrow A \otimes_{C_{0}(T)} C_{0}(T, K)$ respects the identification of spectra we get that

$$
\begin{array}{cll}
\left(A \times_{\gamma} G\right)^{\wedge} & \rightarrow & E \\
p \downarrow & & \downarrow q \\
\hat{A} & = & T
\end{array}
$$

is an isomorphism of $G$-bundles, where $\gamma$ is the coaction of $A$ corresponding to $i \otimes \delta$ under the isomorphism.

6.8. Remark. Combining Theorems 6.7 and 5.11 and Remark 5.12 we see that if $A$ is stable, $\hat{A}$ is paracompact, and $G$ is separable, then the exterior equivalence classes of locally unitary coactions of $G$ on $A$ are in bijective correspondence with the isomorphism classes of locally trivial principal, $G$-bundle over $\hat{A}$.

It would be nice to have a similar result relating (Cartan) principal $G$-bundles and (a suitably restricted class of) pointwise unitary coactions. Some restriction would be needed as one can show that $\left(A \times_{\delta} G\right)^{\wedge}$ need not be Hausdorff, in general. We leave this problem open.

\section{REFERENCES}

1. C. A. Akemann, G. K. Pedersen and J. Tomiyama, Multipliers of $C^{*}$-algebras, J. Funct. Anal. 13 (1973), 277-301.

2. R. J. Archbold and C. J. K. Batty, $C^{*}$-tensor norms and slice maps, J. London Math. Soc. 22 (1980), $127-138$.

3. J. Dixmier, $C^{*}$-algebras, North-Holland, Amsterdam, 1977.

4. P. Eymard, L'algèbre de Fourier d'un groupe localement compact, Bull. Soc. Math. France 92 (1964), 181-236.

5. J. M. G. Fell, Induced representations and Banach *-algebraic bundles, Lecture Notes in Math., vol. 582, Springer-Verlag, Berlin, Heidelberg and New York, 1977.

6. A. M. Gleason, Spaces with a compact Lie group of transformations, Proc. Amer. Math. Soc. 1 (1950), 35-43.

7. P. Green, $C^{*}$-algebras of transformation groups with smooth orbit space, Pacific J. Math. 72 (1977), 71-97.

8. The local structure of twisted covariance algebras, Acta. Math. 140 (1978), 191-250.

9. E. Hewitt and K. A. Ross, Abstract harmonic analysis. Vol. II, Springer-Verlag, Berlin, Heidelberg and New York, 1970.

10. S. Imai and H. Takai, On a duality for $C^{*}$-crossed products by a locally compact group, J. Math. Soc. Japan 30 (1978), 495-504.

11. Y. Katayama, Remarks on a $C^{*}$-dynamical system, Math. Scand. 49 (1981), 250-258.

12. , Takesaki's duality for a nondegenerate coaction, Math. Scand. 55 (1985), 141-151.

13. M. B. Landstad, Duality theory for covariant systems, Trans. Amer. Math. Soc. 248 (1979), 223-267.

14. Y. Nakagami and M. Takesaki, Duality for crossed products of von Neumann algebras, Lecture Notes in Math., vol. 731, Springer-Verlag, Berlin, Heidelberg and New York, 1979.

15. R. S. Palais, On the existence of slices for actions of non-compact Lie groups, Ann. of Math. (2) 73 (1961), 295-323. 
16. G. K. Pedersen, $C^{*}$-algebras and their automorphism groups, Academic Press, London and New York, 1979.

17. J. Phillips and I. Raeburn, Perturbations of $C^{*}$-algebras. II, Proc. London Math. Soc. 43 (1981), $46-72$.

18. , Crossed products by locally unitary automorphism groups and principal bundles, J. Operator Theory 11 (1984), 215-241.

19. J. C. Quigg, Duality for reduced twisted crossed products of $C^{*}$-algebras, Indiana Univ. Math. J. (to appear).

20. I. Raeburn and J. Rosenberg, Crossed products of continuous trace $C^{*}$-algebras by smooth actions, preprint, MSRI, Berkeley, 1985.

21. I. Raeburn and D. P. Williams, Pull-backs of $C^{*}$-algebras and crossed products by certain diagonal actions, Trans. Amer. Math. Soc. 287 (1985), 755-777.

22. H. A. Smith, Commutative twisted group algebras, Trans. Amer. Math. Soc. 197 (1974), 315-326.

23. M. Takesaki, Tomita's theory of modular Hilbert algebras and its applications, Lecture Notes in Math., vol. 128, Springer-Verlag, Berlin, Heidelberg and New York, 1970.

24. __ Duality and von Neumann algebras, Lecture Notes in Math., vol. 247, Springer-Verlag, Berlin, Heidelberg and New York, 1972, pp. 666-779.

25. J. Tomiyama, Applications of Fubini-type theorem to the tensor products of $C^{*}$-algebras, Tôhoku Math. J. 19 (1967), 213-226.

26. S. Wassermann, A pathology in the ideal space of $L(H) \otimes L(H)$, Indiana Univ. Math. J. 27 (1978), 1011-1020.

Department of Mathematics, A. V. H., University of Trondheim, 7055 Dragvoll, Norway

Department of Mathematics, University of Victoria, Victoria, British Columbia, Canada V8W 2 Y2

Department of Mathematics, Dalhousie University, Halifax, Nova Scotia, Canada B3H 4H8

Department of Mathematics, University of New South Wales, Kensington 2033, Australia

Mathematical Sciences Research Institute, Berkeley, California 94720 